MATHEMATICS OF COMPUTATION

Volume 77, Number 261, January 2008, Pages 125-158

S $0025-5718(07) 02008-\mathrm{X}$

Article electronically published on July 26, 2007

\title{
AN INTERPOLATED STOCHASTIC ALGORITHM FOR QUASI-LINEAR PDES
}

\author{
FRANÇOIS DELARUE AND STÉPHANE MENOZZI
}

\begin{abstract}
In this paper, we improve the forward-backward algorithm for quasi-linear PDEs introduced in Delarue and Menozzi (2006). The new discretization scheme takes advantage of the standing regularity properties of the true solution through an interpolation procedure. For the convergence analysis, we also exploit the optimality of the square Gaussian quantization used to approximate the conditional expectations involved.

The resulting bound for the error is closely related to the Hölder exponent of the second order spatial derivatives of the true solution and turns out to be more satisfactory than the one previously established.
\end{abstract}

\section{INTRODUCTION}

1.1. Short overview of numerical schemes for BSDEs. The theory for Backward SDEs (cf. Pardoux and Peng 26] for the original background) nowadays enjoys a new development through numerical applications. As the classical theory introduced during the 90's for backward equations does, the numerical counterpart offers a double panorama: each discretization procedure for BSDEs provides a conceivable scheme for a certain class of non-linear PDEs and vice and versa. Both implications make sense (see e.g. Douglas et al. [10] for a PDE to BSDE approach), but the trend in the current probabilistic literature now consists in exhibiting purely stochastic algorithms for BSDEs and then in deriving alternative methods to analytical finite-difference or finite-element strategies for non-linear PDEs. Of course, this raises the question of the competitiveness of the standing probabilistic methods and draws the objective for the next years: refine as much as possible the earlier algorithms to decrease at most the underlying approximation error and take advantage of the specific stochastic structure to investigate new fields of application (SPDEs, homogenization, etc.).

In this work, we are concerned with non-linear Cauchy problems on $[0, T] \times \mathbb{R}^{d}$ of the following form $\left(\nabla_{x} u\right.$ stands for the $x$-gradient of $u$, seen as a row vector, and $H_{u}$ for the $x$-Hessian matrix of $u$ ):

$$
(\mathcal{E})\left\{\begin{array}{l}
\partial_{t} u(t, x)+\nabla_{x} u(t, x) b(x, u(t, x), v(t, x)) \\
\quad+\frac{1}{2} \operatorname{tr}\left(a(x, u(t, x)) H_{u}(t, x)\right)+f(x, u(t, x), v(t, x))=0, \\
u(T, x)=H(x),
\end{array}\right.
$$

with $v(t, x) \equiv \nabla_{x} u(t, x) \sigma(x, u(t, x))$. The stochastic counterpart of $(\mathcal{E})$ writes as a "fully coupled" Forward Backward Stochastic Differential Equation (FBSDE for

Received by the editor March 30, 2006 and, in revised form, October 31, 2006.

2000 Mathematics Subject Classification. Primary 65C30; Secondary 60H10, 60H35.

(C)2007 American Mathematical Society 125

Reverts to public domain 28 years from publication 
short). Namely, for a given starting point $x_{0} \in \mathbb{R}^{d}$, we consider a diffusion process $U$ strongly coupled to the solution $(V, W)$ of a BSDE by the relation

$$
\forall t \in[0, T],\left\{\begin{array}{l}
U_{t}=x_{0}+\int_{0}^{t} b\left(U_{s}, V_{s}, W_{s}\right) d s+\int_{0}^{t} \sigma\left(U_{s}, V_{s}\right) d B_{s}, \\
V_{t}=H\left(U_{T}\right)+\int_{t}^{T} f\left(U_{s}, V_{s}, W_{s}\right) d s-\int_{t}^{T} W_{s} d B_{s}
\end{array}\right.
$$

where $B$ is a $d$-dimensional Brownian motion on a certain filtered probability space $\left(\Omega,\left(\mathcal{F}_{t}\right)_{t \in[0, T]}, \mathbb{P}\right)$ and $\sigma(x, y)$ a square root of the diffusion matrix $a(x, y)$. For a guided tour of the connection between $(\mathcal{E})$ and $(\mathrm{E})$, we refer the reader to Antonelli [1, Ma, Protter and Yong [20, Ma and Yong [21, Delarue 7] and more recently Delarue and Guatteri 8 . Generally speaking, if $u$ denotes, under suitable assumptions, the solution of $(\mathcal{E})$, the backward component of $(\mathrm{E})$ writes at time $t:\left(V_{t}, W_{t}\right)=\left(u\left(t, U_{t}\right), v\left(t, U_{t}\right)\right)$. Concerning the numerical approximation, we mention the works of Douglas et al. [10] and Milstein and Tretyakov [22, 24] and [23.

Probabilistic algorithms for $(\mathcal{E})$ consist in discretizing the following non-linear form of the dynamic programming principle: $u\left(t, U_{t}\right)=\mathbb{E}\left[u\left(t+h, U_{t+h}\right) \mid \mathcal{F}_{t}\right]+$ $\mathbb{E}\left[\int_{t}^{t+h} f\left(U_{s}, V_{s}, W_{s}\right) d s \mid \mathcal{F}_{t}\right]$.

Decoupled case. In the decoupled case, the forward component can be approximated with a standard Euler scheme so that $V_{t}=u\left(t, U_{t}\right)$ can be reached provided a suitable estimation of $(V, W)$ at time $t+h$.

Once the approximation of $V_{t}$ is available, the next step to iterate the process consists in updating the approximation of the representation process $W$. To this end, one usually uses the so-called martingale increment technique, see e.g. Bally et al. 2 or Bouchard and Touzi 44. Basically, this amounts to saying that $W_{t} \approx$ $h^{-1} \mathbb{E}\left[V_{t+h}\left(B_{t+h}-B_{t}\right) \mid \mathcal{F}_{t}\right]$.

Monte-Carlo techniques are then well fitted to the effective computations of the underlying conditional expectations. Due to the Markov property for $U$ and to the relationship $\left(V_{t}, W_{t}\right)=\left(u\left(t, U_{t}\right), v\left(t, U_{t}\right)\right)$, for $t \in[0, T]$, these latter reduce to conditional expectations with respect to $\sigma$-fields generated by a random vector. Several regression methods are then conceivable: Bouchard and Touzi [4 refer to Malliavin calculus techniques (this involves a rather large number of simulated paths for the underlying diffusion process), and Lemor, Gobet and Warin ([18] and [19]) make use of a finite function basis (this allows them to use the same paths for the approximations of the forward and backward processes).

Coupled case. All the previous methods require an a priori discretized version for the process $U$ and thus fail in our frame, except when considering a global fixed point strategy for the triple $(U, V, W)$ : given a first $U$, compute the associated $(V, W)$, and then plug this $(V, W)$ to compute a new $U$ and so on. We refer to Rivière [27] and Bender and Zhang [3] for first attempts in this direction.

The common strategy in the coupled case relies on spatial grids (see e.g. Delarue and Menozzi 9 and Milstein and Tretyakov [25). At time $t$, the initial condition of the process $U$ in the dynamic programming principle is chosen as a deterministic node $x$ of a Cartesian spatial grid. Given, for a small $h>0$, an approximation $(\bar{u}(t+h, x), \bar{v}(t+h, x))$ of the solution of the PDE and of its gradient at $(t+h, x)$, this permits us to approximate the transition of the diffusion from time $t$ to time $t+h$ and to derive an approximation of $u(t, x)$. The martingale increment technique provides an approximation of the gradient. Such a procedure can be iterated along 
a temporal mesh of step $h$. Underlying expectations are then estimated with a quantization argument that turns out to be cheaper than a Monte-Carlo method.

Anyhow, the approximate transition plugged in the dynamic programming principle is supported by a different set than the grid itself, so that the approximated solution $\bar{u}$ has to be extended from the spatial grid to the whole space. In [9], the considered extension is piecewise constant and thus discontinuous. Here we propose to extend $\bar{u}$ through a piecewise linear interpolation procedure to take the utmost advantage of the standing regularity for the true solution $u$ (see Milstein and Tretyakov 22, 24] and 23] for a similar procedure).

1.2. Contribution and prospects of the paper. The numerical analysis we provide in this paper appears as a new improvement towards competitive probabilistic algorithms for quasi-linear PDEs. As in 22, [24, the global bound we exhibit below (see Theorem 3.2) mainly holds for $b$ independent of $W$ (several extensions to the general frame are discussed in the sequel). However, we feel that it is the first one to apply to both an interpolated stochastic scheme and a classical solution $u \in \mathcal{C}^{1+\alpha / 2,2+\alpha}\left([0, T] \times \mathbb{R}^{d}, \mathbb{R}\right), \alpha$ being possibly small. By way of example, the solutions are required to be at least twice differentiable in time (and therefore four times in space) in the different papers of Milstein and Tretyakov. Generally speaking, the explanation for these different regularity assumptions follows from the error analysis, i.e. from the proofs of the convergence of the underlying algorithm, and not from the very definitions of the algorithms.

Two main conclusions follow from Theorem 3.2, First, as forecasted from purely numerical experiments in [9] and as already proved by Milstein and Tretyakov in the very regular frame, the piecewise linear interpolation procedure reduces the error with respect to the piecewise constant one. We prove here that the gain between both is exactly the one expected. Second, we prove that the algorithm still converges for a low number of points for the underlying quantization of the Brownian increments. In this sense, we recover the results observed in the papers of Milstein and Tretyakov where the Brownian motion is approximated by a simple random walk. We also improve our previous work in which quantization is assumed to be "large" enough to ensure the convergence.

The case where $b$ depends on $W$ is crucial for applications: the so-called deterministic KPZ equations, i.e. the heat equation forced by the square norm of the gradient of the solution, both appear in statistical mechanics (see e.g. Woyczyǹski [29]) and in finance (see e.g. Hu et al. [13]). In the case of smooth coefficients (and in particular for a bounded $b$ ), a suitable probabilistic algorithm and the associated convergence analysis can be found in 23], Section 3 . In our setting, we can treat the case where $b$ is bounded as a zero drift situation with $f(x, u, v)+v \sigma^{-1}(x, u) b(x, u, v)$ as the second member. In the general framework (that is, for unbounded drifts), we just manage to establish the convergence of the algorithm provided the quantization is large enough (see Section 7). Even if not completely satisfactory, this result is, to the best of our knowledge, new in the probabilistic literature devoted to the subject. For $b$ with a linear growth in $v$, we also show that the interpretation of the product $v \sigma^{-1}(x, u) b(x, u, v)$ as a part of the second member term, with a possible quadratic growth in $v$, may fail from a numerical point of view.

Beyond these remarks, several questions are to be investigated in future contributions. First, the interpolation procedure we consider here is well fitted to our own setting since the Lagrange kernel of order one can be interpreted as a family 
of probability weights. This fails for higher order kernels so that the interest of an interpolation procedure of order two remains open. Second, the real influence of the quantization in the low regular framework (i.e. for $\alpha$ close to zero) is rather subtle to analyze in light of numerical experiments and is to be understood. Indeed, for a solution with isolated "singularities" (i.e. a solution for which $H_{u}$ is smooth except in several points), the error observed in various examples may vary with the number of points for the quantization.

Finally, our analysis of the algorithm provides a possible discretization procedure for the FBSDE (E). For systems driven by Lipschitz continuous coefficients, this discretization turns out to be strongly convergent, as already shown in our previous paper. In the case of space Hölder continuous coefficients, as it may be under our assumptions, the error has to be analyzed in the weak sense. We don't investigate this point in the current work.

1.3. Organization of the paper. In Section2 we state our working assumptions. In Section 3, we introduce the interpolated algorithm and the associated convergence result. Section 4 is dedicated to numerical illustrations. The proofs of the main results are given in the remaining parts of the paper. Section 7 is specifically devoted to the case $b=b(x, u, v)$.

\section{WORKING ASSUMPTIONS AND ASSOCIATED PROPERTIES}

For a given $d \in \mathbb{N}^{*}$, we consider the coefficients $b: \mathbb{R}^{d} \times \mathbb{R} \times \mathbb{R}^{d} \rightarrow \mathbb{R}^{d}, f$ : $\mathbb{R}^{d} \times \mathbb{R} \times \mathbb{R}^{d} \rightarrow \mathbb{R}, \sigma: \mathbb{R}^{d} \times \mathbb{R} \rightarrow \mathbb{R}^{d \times d}, H: \mathbb{R}^{d} \rightarrow \mathbb{R}$

Assumption (A). The functions $b, f, H$ and $\sigma$ are said to satisfy Assumption (A) if they are bounded in space, have at most linear growth in the other variables, and are uniformly $\alpha$-Hölder continuous in $x, \alpha>0$, and uniformly Lipschitz continuous w.r.t. the other variables, if $a \equiv \sigma \sigma^{*}$ is uniformly elliptic and if $H$ is bounded in $C^{2+\alpha}\left(\mathbb{R}^{d}\right)$.

From now on, Assumption (A) is in force. We denote by $|\cdot|$ the Euclidean norm of $\mathbb{R}^{d}$, and by $\langle\cdot, \cdot\rangle$ the associated inner product.

2.1. Forward-backward SDE. Now consider a given $T>0$ and an initial condition $x_{0} \in \mathbb{R}^{d}$. According to Delarue and Guatteri 8, there exists a filtered probability space $\left(\Omega,\left(\mathcal{F}_{t}\right)_{0 \leq t \leq T}, \mathbb{P}\right)$ endowed with a $d$-dimensional Brownian motion $\left(B_{t}\right)_{0 \leq t \leq T}$ as well as a progressively measurable triple $(U, V, W)$, with values in $\mathbb{R}^{d} \times \mathbb{R} \times \mathbb{R}^{d}$, such that $\mathbb{E} \sup _{t \in[0, T]}\left(\left|U_{t}\right|^{2}+\left|V_{t}\right|^{2}\right)<+\infty, \mathbb{E} \int_{0}^{T}\left|W_{t}\right|^{2} d t<+\infty$, and which satisfies $\mathbb{P}$ almost surely the equations $(\mathrm{E})$. The distribution of the fourtuple $(B, U, V, W)$ is unique on the space $\mathcal{C}\left([0, T], \mathbb{R}^{2 d+1}\right) \times L^{2}\left([0, T], \mathbb{R}^{d}\right)$. In other words, the FBSDE (E) admits a unique weak solution. For $\alpha=1$, existence and uniqueness hold in a strong sense.

2.2. Quasi-linear PDE. According to Ladyzhenskaya et al. [17, Ch. 7, Th 7.1] and to 20 (up to a regularization procedure of the coefficients), we claim that $(\mathcal{E})$ admits a solution $u \in \mathcal{C}^{1,2}\left([0, T] \times \mathbb{R}^{d}, \mathbb{R}\right)$ satisfying: 
Theorem 2.1. There exists a constant $C_{[2.1}$, depending only on $T$ and on known parameters appearing in $\mathbf{( A )}$, such that $\forall(t, x) \in[0, T] \times \mathbb{R}^{d}$,

$$
\begin{aligned}
|u(t, x)| & +\left|\nabla_{x} u(t, x)\right|+\left|\nabla_{x, x}^{2} u(t, x)\right|+\left|\partial_{t} u(t, x)\right| \\
& +\sup _{t^{\prime} \in[0, T], t \neq t^{\prime}}\left[\left|t-t^{\prime}\right|^{-(1+\alpha) / 2}\left|\nabla u(t, x)-\nabla u\left(t^{\prime}, x\right)\right|\right] \\
& +\sup _{x^{\prime} \in \mathbb{R}^{d}, x \neq x^{\prime}}\left[\left|x-x^{\prime}\right|^{-\alpha}\left|\nabla_{x, x}^{2} u(t, x)-\nabla_{x, x}^{2} u\left(t, x^{\prime}\right)\right|\right] \leq C_{2.1} .
\end{aligned}
$$

Moreover, $u$ is unique in the class of functions

$$
\widetilde{u} \in \mathcal{C}\left([0, T] \times \mathbb{R}^{d}, \mathbb{R}\right) \cap \mathcal{C}^{1,2}\left(\left[0, T\left[\times \mathbb{R}^{d}, \mathbb{R}\right)\right.\right.
$$

for which $\sup _{(t, x) \in\left[0, T\left[\times \mathbb{R}^{d}\right.\right.}\left(|\widetilde{u}(t, x)|+\left|\nabla_{x} \widetilde{u}(t, x)\right|\right)<+\infty$.

The connection between $(\mathrm{E})$ and $(\mathcal{E})$ can be summarized as follows:

$$
\left(V_{t}, W_{t}\right)=(u, v)\left(t, U_{t}\right), V_{t}=\mathbb{E}\left[V_{T} \mid \mathcal{F}_{t}\right]+\mathbb{E}\left[\int_{t}^{T} f\left(U_{s}, V_{s}, W_{s}\right) d s \mid \mathcal{F}_{t}\right] .
$$

\section{Algorithm And main Results}

Following Delarue and Menozzi [9], we now introduce the basic objects for the discretization procedure of $(\mathrm{E})$ and $(\mathcal{E})$, namely a temporal mesh as well as a family of spatial grids and an optimal quantization for the Gaussian law. In addition to these ingredients, we consider a collection of interpolating functions associated to the underlying spatial grids.

3.1. Construction of the interpolated algorithm. For clarity reasons, we choose to define the approximated solution on a family of infinite spatial grids. This is not realistic from a purely numerical point of view, anyhow the truncation procedure is highly discussed in Delarue and Menozzi 9]. It induces heavy computations for the error analysis and is totally useless for our original purpose. For this reason, we consider $\mathcal{C}_{\infty} \equiv \delta \mathbb{Z}^{d}, \delta>0$, the infinite Cartesian grid of step $\delta$.

Shape functions. The algorithm we propose below is based on a piecewise multilinear approximation procedure, obtained by tensorization of piecewise linear interpolation. The involved $[0,1]$-valued shape functions are the following:

$$
\forall z \in \mathcal{C}_{\infty}, \forall x \in \mathbb{R}^{d}, \phi_{z}(x)=\prod_{i=1}^{d} \Phi\left(\delta^{-1}\left(x_{i}-z_{i}\right)\right),
$$

with $\Phi(t)=(1-|t|)_{+}$. Obviously, for $z \in \mathcal{C}_{\infty}, \phi_{z}$ is non-negative, is equal to one in $x=z$ and vanishes outside the hypercube centered at $z$ having edge lengths $2 \delta$. It is plain to see that such a family interpolates exactly polynomials of order less than one:

$$
\forall x \in \mathbb{R}^{d}, \sum_{z \in \mathcal{C}_{\infty}} \phi_{z}(x)=1, \sum_{z \in \mathcal{C}_{\infty}} \phi_{z}(x) z=x .
$$

We refer the reader to the literature devoted to finite elements (see e.g. Brenner and Scott [5]) for more general examples of shape functions. Anyhow, due to the stochastic interpretation of the algorithm we provide below, we are to view the underlying family of shape functions in terms of probability weights. Hence, the method is valid only for non-negative shape functions with sum equal to 1 . This prevents us from introducing Lagrange kernels of order greater than two since they 
may take negative values (see the monograph of Milstein and Tretyakov [25, p. 425] for similar remarks). In the sequel, we denote for a given function $\psi: \mathcal{C}_{\infty} \rightarrow \mathbb{R}$

$$
\forall x \in \mathbb{R}^{d}, \psi_{\delta}(x)=\sum_{z \in \mathcal{C}_{\infty}} \phi_{z}(x) \psi(z),
$$

its interpolation associated to the sequence $\left(\phi_{z}\right)_{z \in \mathcal{C}_{\infty}}$. Note that for $z \in \mathcal{C}_{\infty}, \psi(z)=$ $\psi_{\delta}(z)$.

Time mesh. Finally, let us introduce a uniform time mesh of $[0, T]$ with time step $h>0, h=T / N, N \in \mathbb{N}^{*}$, i.e. $\left\{\left(t_{i} \equiv i h\right)_{i \in \llbracket 0, N \rrbracket}\right\}$.

3.2. Algorithm. In the spirit of Delarue and Menozzi [9], we define

\section{Algorithm 3.1.}

$$
\begin{aligned}
& \forall x \in \mathbb{R}^{d}, \bar{u}(T, x) \equiv H(x), \bar{v}(T, x) \equiv \nabla_{x} H(x) \sigma(x, H(x)), \\
& \forall k \in \llbracket 0, N-1 \rrbracket, \forall x \in \mathcal{C}_{\infty}, \\
& \\
& \mathcal{T}\left(t_{k}, x\right) \equiv b\left(x, \bar{u}\left(t_{k+1}, x\right), \bar{v}\left(t_{k+1}, x\right)\right) h+\sigma\left(x, \bar{u}\left(t_{k+1}, x\right)\right) \gamma^{*} g\left(\Delta B^{k}\right), \\
& \quad \bar{v}\left(t_{k}, x\right) \equiv h^{-1} \mathbb{E}\left[\bar{u}_{\delta}\left(t_{k+1}, x+\mathcal{T}\left(t_{k}, x\right)\right) g\left(\Delta B^{k}\right)^{*}\right] \gamma, \\
& \quad \bar{u}\left(t_{k}, x\right) \equiv \mathbb{E}\left[\bar{u}_{\delta}\left(t_{k+1}, x+\mathcal{T}\left(t_{k}, x\right)\right)\right]+h f\left(x, \bar{u}\left(t_{k+1}, x\right), \bar{v}\left(t_{k}, x\right)\right) .
\end{aligned}
$$

In the above algorithm, $\Delta B^{k} \equiv B_{t_{k+1}}-B_{t_{k}}$ where $\left(\left(B_{t}\right)_{t \geq 0},\left(\mathcal{F}_{t}^{B}\right)_{t \geq 0}\right)$ denotes a $d$-dimensional Brownian motion and its natural augmented filtration on a probability space $(\Omega, \mathcal{F}, \mathbb{P})$. Also, $g\left(\Delta B_{k}\right) \equiv h^{1 / 2} \Pi_{M}\left(h^{-1 / 2} \Delta B^{k}\right), \Pi_{M}$ being the projection mapping onto a square optimal quantization grid $\Lambda_{M}^{*}$, with $M$ points, for the $d$ dimensional standard Gaussian vector. In other words, integrals with respect to the Gaussian kernel are replaced by discrete sums that turn out to be numerically computable. The only controls concerning the quantized Brownian increment that will be needed in the sequel are the following:

$$
\begin{aligned}
& \mathbb{E}\left[\left|g\left(\Delta B^{k}\right)-\Delta B^{k}\right|^{2}\right] \leq C_{\text {Quantiz }}(d) h M^{-2 / d}, \\
& \mathbb{E}\left[\Delta B^{k} \mid g\left(\Delta B^{k}\right)\right]=g\left(\Delta B^{k}\right) .
\end{aligned}
$$

Note that the last property simply expresses that the quantized variable is a projector. It also implies $\mathbb{E}\left[g\left(\Delta B^{k}\right)\right]=0$. For details about quantization, we refer to the monograph of Graf and Luschgy [12].

The reader who is used to stochastic literature may wonder why we do not employ a Monte-Carlo strategy. The reason can be explained as follows: for a while replace $\bar{u}_{\delta}\left(t_{k+1}, \cdot\right)$ by the true solution $u\left(t_{k+1}, \cdot\right)$ in the above induction. Since the latter function belongs to $\mathcal{C}^{2+\alpha}\left(\mathbb{R}^{d}\right)$, the quantization procedure then provides a better approximation for the integral with respect to the Gaussian kernel than the MonteCarlo one, that is known to be well fitted to rough frameworks.

Finally detail the meaning of $\gamma$ in Algorithm 3.1. Denote the covariance matrix of the quantized $d$-dimensional standard Gaussian law by

$$
K_{M} \equiv \mathbb{E}\left[\Pi_{M}\left(\mathcal{N}\left(0, I_{d}\right)\right) \times \Pi_{M}\left(\mathcal{N}\left(0, I_{d}\right)\right)^{*}\right] .
$$

$\gamma$ then stands for the lower triangular matrix of the Cholesky writing of $K_{M}^{-1}$, i.e. $\gamma \gamma^{*}=K_{M}^{-1}$ (provided $\operatorname{det}\left(K_{M}\right)>0$ ). The introduction of $\gamma$ in the algorithm follows from the same trick as in the former paragraph: for a while replace $\bar{u}\left(t_{k+1}, \cdot\right)$ and $\bar{u}_{\delta}\left(t_{k+1}, \cdot\right)$ by $u\left(t_{k+1}, \cdot\right)$ both in the local transition $\mathcal{T}\left(t_{k}, x\right)$ and in the definition of $\bar{v}\left(t_{k}, x\right)$ and focus on the resulting martingale increment 
$h^{-1} \mathbb{E}\left[u\left(t_{k+1}, x+\mathcal{T}\left(t_{k}, x\right)\right) g\left(\Delta B^{k}\right)^{*}\right] \gamma$. Since the true solution is smooth, the Taylor expansion yields as its first approximation:

$$
\begin{aligned}
h^{-1} \mathbb{E}\left[u\left(t_{k+1}, x+\mathcal{T}\left(t_{k}, x\right)\right)\right. & \left.g\left(\Delta B^{k}\right)^{*}\right] \gamma \\
& \sim \nabla_{x} u\left(t_{k+1}, x\right) \sigma\left(x, u\left(t_{k+1}, x\right)\right) \gamma^{*} K_{M} \gamma=v\left(t_{k+1}, x\right) .
\end{aligned}
$$

When corrected by $\gamma$, the martingale increment associated to a smooth function is worth, up to negligible terms, the gradient of the underlying function multiplied by the diffusion coefficient of the transition.

In the sequel, we also write the coefficients of the transition $\mathcal{T}\left(t_{k}, x\right)$ in the following abbreviated way: $\mathcal{T}\left(t_{k}, x\right) \equiv \beta\left(t_{k}, x\right) h+\Sigma\left(t_{k}, x\right) \gamma^{*} g\left(\Delta B^{k}\right)$ with $\beta\left(t_{k}, x\right) \equiv$ $b\left(x, \bar{u}\left(t_{k+1}, x\right), \bar{v}\left(t_{k+1}, x\right)\right), \Sigma\left(t_{k}, x\right) \equiv \sigma\left(x, \bar{u}\left(t_{k+1}, x\right)\right) \gamma^{*}$.

\subsection{Main results.}

Theorem 3.2. Assume that $b$ does not depend on $v$. Then, there exist two constants $4 \overline{3.2}>0$ and $C_{\overline{3.2}}$, only depending on $T$ and on known parameters appearing in $(\mathbf{A})$, such that for $h<4$ [3.2:

$$
\begin{aligned}
& \sup _{x \in \mathbb{R}^{d}, k \in \llbracket 0, N \rrbracket}\left|u\left(t_{k}, x\right)-\bar{u}_{\delta}\left(t_{k}, x\right)\right|^{2} \leq C_{3.2}\left[\mathcal{E}^{2} \text { (time) }+\mathcal{E}^{2} \text { (space) }+\mathcal{E}^{2} \text { (quantiz) }\right] \\
& \equiv C_{3.2} \mathcal{E}^{2} \text { (global) } \\
& \text { with } \mathcal{E} \text { (time) } \equiv h^{\alpha / 2}, \mathcal{E} \text { (space) } \equiv h^{-1} \delta^{2} \text {, and } \mathcal{E} \text { (quantiz) } \equiv h^{\alpha / 2} M^{-2 / d} \text {. }
\end{aligned}
$$

\section{Classification of the errors.}

Time error. This term results from the combination of the $1 / 2$-Hölder continuity of the Brownian motion in the $L^{2}$ sense and of the $\alpha$-Hölder $x$-continuity of the coefficients of the PDE.

Formally, for $\alpha=2$, we recover the rate announced in the papers of Milstein and Tretyakov.

Spatial error. The distance between a smooth function (think of the true solution) and its piecewise linear interpolated version at the nodes of the grid is worth $\delta^{2}$. Due to the propagation of the error along the time mesh, the resulting spatial error is $h^{-1} \delta^{2}$. Compared to the rough projection in [9], i.e. piecewise constant interpolation, we gain one order w.r.t. $\delta$. This is the expected improvement with such a procedure, and the new bound is in this sense satisfactory.

The reader may wonder why we restrict to an interpolation of order one since $u$ is $C^{2+\alpha}$ in space. The reason follows once again from the probabilistic nature of the algorithm that prevents us, at least in terms of convergence analysis, from using negative weights deriving from higher order interpolators.

From the structure of the spatial error we derive that $\delta$ has to be small against $h$. Indeed, the spatial grid has to be fine enough to catch the increments of the Brownian motion. This ratio is the inverse of the one needed for the stability of a deterministic algorithm for second order parabolic PDEs.

Quantization error. For a bounded smooth function $F \in C_{b}^{2}\left(\mathbb{R}^{d}, \mathbb{R}\right)$ (i.e. with bounded derivatives of order one and two) and an optimally quantized Gaussian kernel, we have from (3.4) : $\mathbb{E}[F(\Delta B)]-\mathbb{E}[F(g(\Delta B))]=O\left(h M^{-2 / d}\right)$. The term $M^{-2 / d}$ is obtained summing along the mesh. The $h^{\alpha / 2}$ corresponds to the Hölder regularity of $H_{u}$ and appears through some rather sharp controls during the error analysis. 
Influence of the quantization. In comparison with Delarue and Menozzi [9, this new bound for $\mathcal{E}$ (quantiz) is rather spectacular. The reason is the following: we take advantage of the optimality of the quantization; see (3.5). On the opposite side, the quantization in 9] is not assumed to be optimal since the analysis relies on $L^{p}$ bounds for the underlying Gaussian quantization, that are known to fail in the optimal setting (see the recent paper of Graf, Luschgy and Pagès [11]).

The crucial point, in our new frame, is the following: the quantization error is less than the temporal one. In particular, there does not seem to be any interest to choose a large support for the quantized Gaussian kernel: it is sufficient to choose one of the roughest quantization grids satisfying $\operatorname{det}\left(K_{M}\right)>0$. Actually, this phenomenon is not so surprising: in the one-dimensional smooth case investigated by Milstein and Tretyakov [25, that is, $\alpha=2$ in a formal way, the global error is worth $h$ provided $\delta=h$ and $M=2$ (in fact, the latter authors do not refer to the quantization theory, but directly approximate the Gaussian law by a centered Bernoulli one).

Paradoxically, we feel that a large quantization may be useful in the non-smooth setting. Indeed, for $\alpha$ close to zero, the order of the expected global error cannot exceed $\alpha / 2$, due to the temporal error $\mathcal{E}$ (time), and is thus very low. On the opposite side, the terms $\mathcal{E}$ (space) and $\mathcal{E}$ (quantiz) can be chosen of order $1 / 2$ provided $\delta=$ $h^{3 / 4}$ and $M=h^{-d / 4}$ (such values are still reasonable in the three-dimensional setting). The question is then the following: how do we diminish $\mathcal{E}$ (time)?

As explained above, the bound for $\mathcal{E}$ (time) follows from the smoothness of the coefficients. To understand exactly what happens, focus on a simple semilinear case: assume that $b$ vanishes and that $\sigma$ reduces to identity. Generally speaking, the underlying strategy of Algorithm 3.1 then consists in approximating $\left.\mathbb{E} \int_{t_{k}}^{t_{k+1}} f\left(B_{s}, u\left(s, B_{s}\right), v\left(s, B_{s}\right)\right) d s, k \in \llbracket 0, N\right)$, by $h \mathbb{E} f\left(B_{t_{k}}, \bar{u}_{\delta}\left(t_{k}, B_{t_{k}}\right), \bar{v}_{\delta}\left(t_{k}, B_{t_{k}}\right)\right)$. If $f$ is just Hölder continuous in $x$, with respect to a small Hölder exponent, we cannot expect to recover less than $h^{\alpha / 2}$ for the temporal error. However, we could approximate $\mathbb{E} \int_{t_{k}}^{t_{k+1}} f\left(B_{s}, u\left(s, B_{s}\right), v\left(s, B_{s}\right)\right) d s$ by $\mathbb{E} \int_{t_{k}}^{t_{k+1}} f\left(B_{s}, \bar{u}_{\delta}\left(t_{k}, B_{t_{k}}\right)\right.$, $\left.\bar{v}_{\delta}\left(t_{k}, B_{t_{k}}\right)\right) d s$ with a Monte-Carlo method or a quantization procedure depending on the exact value of $\alpha$ and the affordable complexity. Even if numerically heavy, the modification of Algorithm 3.1 based on this Monte-Carlo method would provide a convergent scheme in the limit case $\alpha=0$.

Another phenomenon may occur for small values of $\alpha$. The coefficients and the solution may count isolated singularities (that is, isolated points at which $\alpha$ is actually tiny) and have, elsewhere, large "pockets" of smoothness. Such a case is very difficult to investigate from a theoretical point of view. Anyhow, we observe in practice (see Section 4 below) that quantization may be, in some cases, more efficient for large values of $M$. A possible explanation is the following: there may be a competition between the bounds for the global and local errors. In other words, the error is, away from the singularities, of order one, and, around them, of order $\alpha / 2$, and the combination of both is sensible to the value of $M$.

Typical values. In dimensions two and three (that is, in the cases considered in Section (4), numerical computations show that $\operatorname{det}\left(K_{M}\right)>0$ for $M \geq 2^{d}$. We believe the result to be true for higher values of $d$, but the proof remains open. For this choice of $M$ and for $\delta=h^{1 / 2+\alpha / 4}$, the error is at most of order $\alpha / 2$.

What about a gradient dependence in the drift? Compared to 9, there is no $\mathcal{E}$ (gradient) term in the writing of $\mathcal{E}$ (global). The reason is simple: we just 
focus in the current setting on the case $b(x, u)$, whereas $\mathcal{E}$ (gradient) appears when considering the more general case $b(x, u, v)$.

The first strategy to handle the case $b(x, u, v)$ is the following: the drift term can always be seen as a part of the second member. This amounts to considering $g(x, u, v)=f(x, u, v)+v \sigma^{-1}(x, u) b(x, u, v)$ instead of $f(x, u, v)$ itself. Of course, this new coefficient $g$ doesn't satisfy Assumption (A) since it may be of quadratic growth in $v$, and as a matter of fact, locally Lipschitz continuous.

Anyhow, if the drift $b$ is assumed to be bounded, then the function $g$ is at most of linear growth and satisfies for a suitable constant $C \geq 0$ and for all $x, x^{\prime}, z, z^{\prime} \in \mathbb{R}^{d}$ and $y, y^{\prime} \in \mathbb{R}$,

$$
\left|g(x, y, z)-g\left(x^{\prime}, y^{\prime}, z^{\prime}\right)\right| \leq C(1+|z|)\left(\left|x-x^{\prime}\right|^{\alpha}+\left|y-y^{\prime}\right|+\left|z-z^{\prime}\right|\right) .
$$

As explained in Section 7 , we are then able to take advantage of the boundedness of the gradient of the true solution (see Theorem 2.1) to prove that Theorem 3.2 still holds when applied to the four-tuple $(0, g, H, \sigma)$ (i.e. to the equation $(\mathcal{E})$ with zero as drift term and $g$ as its source term). This strategy corresponds to the exponential change of probability performed in [23, Sec. 3] in a similar setting (choose $c=f=0$ there in order to recover our own framework).

It is well understood that the story is rather different when $b$ is not bounded, and therefore, $g$ is of quadratic growth in $\nabla_{x} u$. From a theoretical point of view, quadratic (F)BSDEs are investigated with a suitable exponential transform that is highly discussed in Kobylanski [16]. The possible adaptation of this transform to the discretization procedure is formally open, but the application of Algorithm 3.1 to the quadratic framework, for example to the KPZ equation, provides disappointing numerical results.

The KPZ equation is the heat equation driven by a non-linear term of the form $\left|\nabla_{x} u\right|^{2}$ (the original terminology is inherited from the model of mechanical statistics by Kardar et al. [15], where the equation is additionally forced by a white noise). This equation frequently appears in mathematical finance (think to utility maximization, see e.g. $\mathrm{Hu}$ et al. [13]) and provides a very interesting numerical example. The non-linearity $\left|\nabla_{x} u\right|^{2}$ can be both interpreted as a quadratic second member, so that the drift reduces to zero, or as a first order term with a non-linear drift given by the gradient itself, so that the second member vanishes. We show in Section 4 that the numerical counterpart of the first writing may be unstable and may even explode. On the opposite side, the second point of view yields a good approximation of the true solution.

This is the reason why the case $b(x, u, v)$ is so important to investigate. As is easily guessed by the reader, we are not able to prove the convergence of Algorithm 3.1 in this larger setting. A possible solution is discussed in Section 7 it consists in introducing an intermediate predictor for the gradient in the drift of the approximate transition $\mathcal{T}\left(t_{k}, x\right)$, but induces a new error term, denoted in Delarue and Menozzi [9] by $\mathcal{E}$ (gradient). The new bound for the global error remains the same as in Theorem 3.2, up to a new constant $C_{3.2,}$, provided $\delta^{2} \leq h$ and $\delta^{-3} h^{3 / 2} M^{-2 / d} \leq 1$. Of course, this condition is not satisfactory since $\delta^{-3} h^{3 / 2} M^{-2 / d}$ is worth $h^{-3 \alpha / 4} / 4$ for the above typical values. To recover the order $\alpha / 2, M$ has to be chosen equal to $h^{-3 d \alpha / 8}$. This is the best we can do so far from a theoretical point of view.

Anyhow, the condition we impose on $M$ seems to be useless in the numerical experiments we provide below. For example, we investigate in Subsection 4.2 the $\mathrm{KPZ}$ equation in dimension three with a smooth initial condition (i.e. $\alpha=2$ in a 
formal way). For $\delta$ and $h$ of the same order, we still observe an error of order one w.r.t. $h$, although $M$ is equal to 8 .

What about a truncation? Similarly to what has been done in 9], one could truncate the grid and obtain, for every $q \geq 1$, a truncation error $\mathcal{E}$ (trunc) $\leq$ $C_{q}(R /(R+\rho))^{q}$ where $R>0$ is the radius of the initial grid $\mathcal{C}_{0}$ and $\rho>0$ a truncation parameter. The constant $C_{q}$ blows up when $q$ increases.

\section{NumERICAL EXAMPLES}

In this section, we choose to illustrate various behaviors of the algorithm through the approximation of the multidimensional Burgers equations and the deterministic KPZ equation.

4.1. Multidimensional Burgers equations. The Burgers equations are a simplified form of the Navier-Stokes equations. The convective and dissipative parts are the same, but the pressure term as well as the incompressibility constraint are neglected. The equations write

$$
\begin{aligned}
& \partial_{t} u-\left(u . \nabla_{x}\right) u+\frac{\varepsilon^{2}}{2} \Delta u=0,(t, x) \in[0, T) \times \mathbb{R}^{d}, \varepsilon>0, \\
& u(T, x)=H(x), x \in \mathbb{R}^{d},
\end{aligned}
$$

where $\forall i \in \llbracket 1, d \rrbracket,\left(\left(u . \nabla_{x}\right) u\right)_{i}=\nabla_{x} u_{i} \times u$. Even though the convergence results are stated for real valued functions, the same analysis could be carried out for systems of equations. Thus, Theorem 3.2 is still valid for the solution of (4.1).

In dimension one, it is well known that equation (4.1) has an explicit solution obtained through a Cole-Hopf factorization; see e.g. 29. In the multidimensional setting, the factorization can be done provided the final condition $H$ derives from a potential, namely $H=\nabla H_{0}$, where $H_{0}$ is a real-valued function. In this case, the solution explicitly writes: $\forall(t, x) \in[0, T] \times \mathbb{R}^{d}$,

$$
u(t, x)=\frac{\mathbb{E}\left[\nabla H_{0}\left(x+\varepsilon B_{T-t}\right) \exp \left(-\varepsilon^{-2} H_{0}\left(x+\varepsilon B_{T-t}\right)\right)\right]}{\mathbb{E}\left[\exp \left(-\varepsilon^{-2} H_{0}\left(x+\varepsilon B_{T-t}\right)\right)\right]} .
$$

We always consider the coupled interpretation of the Burgers equations, i.e. $b(x, u)=u$ and $f=0$. This choice turns out to be numerically more robust; see Section 4.2 and also [9], Section 5. In the following, we take $d=2$.

Now discuss the influence of the viscosity parameter. The approximated transitions involved in Algorithm 3.1 are close to $\varepsilon h^{1 / 2}$. To catch them, the spatial grid has to be fine enough and the spatial step $\delta$ has to be, at least, less than $\varepsilon h^{1 / 2}$. This empirical condition is confirmed from a numerical point of view. We thus choose the following values for the parameters at hand: $T=3 / 8, h=2.5 \times 10^{-2}, \delta=$ $.01, \varepsilon^{2}=.4$.

To avoid truncation problems, we choose the periodic initial solution $H_{0}(x)=$ $\prod_{i=1,2} \sin ^{2}\left(\pi x_{i}\right)$. Since the problem is then symmetric, we only present the results obtained for the approximation of the first component of $u=\left(u_{1}, u_{2}\right)^{*}$. We first plot the profiles of the solution $u_{1}$ at $t=0$ and the pointwise absolute error between the reference value and the approximation deriving from Algorithm 3.1, both with and without $\gamma^{*}$. We also investigate the influence of the number $M$ of points used for the quantization (we first choose $M=4$ and then $M=150$ ). The reference value is obtained through the explicit representation (4.2) via quantization with 
600 points: due to the "large" viscosity, we observe on the left top figure how fast the solution decays.
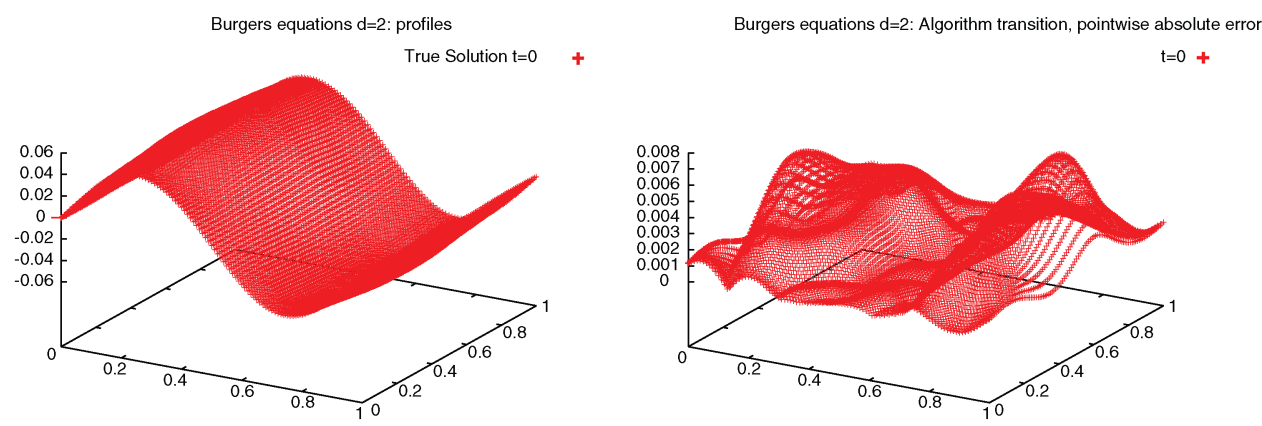

Burgers equations $d=2$ : "Natural" transition, pointwise absolute erro

Burgers equations $d=2$ : pointwise absolute difference between $M=4$ and $M=150$

$\mathrm{t}=0+$

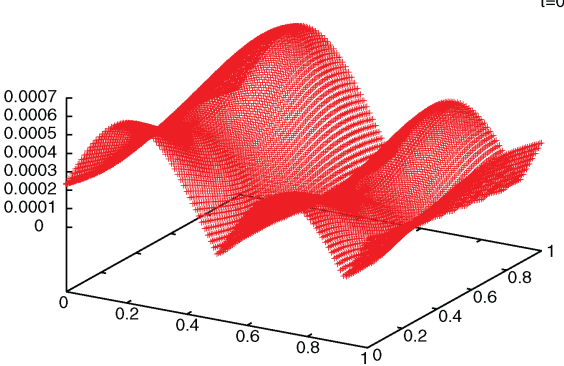

The left bottom figure illustrates, in comparison with the right top one, how the corrector matrix $\gamma^{*}$ in the transition is crucial, especially when the number of points in the quantization is small: with $M=4$, the relative error is close to 2 for $\gamma=I_{d}$, but close to .1 for the suitable $\gamma$ (in this latter case, the relative error is still "large" due to the tiny values of the true solution). Moreover, the last picture confirms that increasing $M$ does not improve the error.

Let us turn to the case of a smaller viscosity, namely $\varepsilon^{2}=.08$. For the previous example, with $M=4, T=.5$, let $\delta$ vary as $\varepsilon h$. The reference solution has globally the same shape as in the previous picture and is $[-.4, .4]$ valued. We obtain

\begin{tabular}{|c|c|c|c|c|}
\hline$h$ & $5 \times 10^{-2}$ & $2.5 \times 10^{-2}$ & $1.25 \times 10^{-2}$ & $6.25 \times 10^{-3}$ \\
\hline $\sup _{t_{i}, x_{j}}\left|\left(\bar{u}_{1}-u_{1}\right)\left(t_{i}, x_{j}\right)\right|$ & .1218 & .0628 & .0439 & .0356 \\
\hline $\sup _{t_{i}, x_{j}}\left|\left(\bar{u}_{2}-u_{2}\right)\left(t_{i}, x_{j}\right)\right|$ & .1215 & .0624 & .0437 & .0355 \\
\hline
\end{tabular}

These numerical results confirm that in the "smooth" case the error is of order one w.r.t. $h$. Note also that the errors for $u_{1}$ and $u_{2}$ differ (whereas the problem is symmetrical) because the optimally quantized grid with 4 points is not isotropic.

4.2. KPZ equation. The KPZ equation can be seen as the primitive of the former Burgers equations. It writes:

$$
\begin{aligned}
& \partial_{t} u+\frac{1}{2}\left|\nabla_{x} u\right|^{2}+\frac{1}{2} \Delta u=0,(t, x) \in[0, T) \times \mathbb{R}^{d}, \\
& u(T, x)=H(x), x \in \mathbb{R}^{d} .
\end{aligned}
$$


Equation (4.3) also has an explicit solution obtained through a Cole-Hopf factorization

$$
u(t, x)=\log \left(\mathbb{E}\left[\exp \left(H\left(x+B_{T-t}\right)\right)\right]\right) .
$$

We first concentrate on the 3 -dimensional case taking

$$
H(x)=10 \exp \left(-(1 / 8)\left(3 x_{1}^{2}+2 x_{2}^{2}+x_{3}^{2}\right)\right), T=.5, h=.02, \delta=.025, M=8 .
$$

We truncate the grid on $[-3,3]$. In the decoupled case, i.e., the underlying process is the Brownian motion, $b=0, f(x, y, z)=\frac{1}{2}|z|^{2}$, and exponential bounds are available for the truncation error through Bernstein-like arguments. In the coupled case, i.e. $b(x, y, z)=\frac{1}{2} z, f=0$, since we do not prove the boundedness of the approximated gradient we only have polynomial controls; see 9]. Anyhow, the fast decay of the terminal condition makes the associated error "numerically" reasonable.

The reference value was computed by quantization from (4.4) with $M=200$. We plot the difference between the reference value and the coupled algorithm. In this smooth case, we still observe an error of order one w.r.t. $h$.
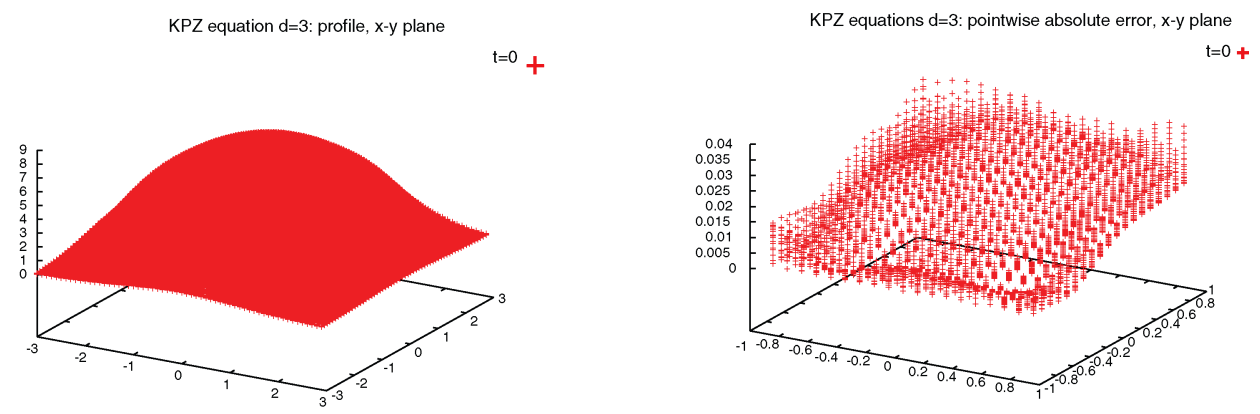

Let us now turn to an example for which the approximated solution obtained with the decoupled algorithm explodes. Take $d=2, H(x)=10 \cos \left(5|x|^{2}\right), T=$ $.1, h=.02, \delta=.02, M=4$ and truncate the grid for $|x| \geq 1$. The reference value is still computed by quantization with $M=200$. One gets
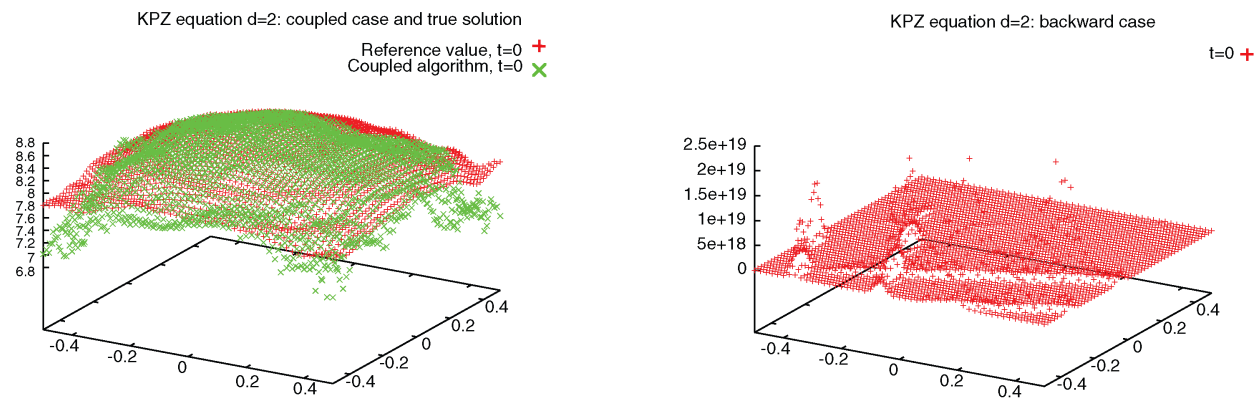

Basically, the numerical integration of a large gradient induces overflows. Hence, for highly oscillating initial data, the coupled interpretation has to be preferred to the backward one that is quite unstable. 
4.3. A toy example in the low regular setting. To illustrate the behavior of the algorithm for small values of $\alpha$, we investigate a linear example. Of course, our algorithm is totally useless in this frame, but reference values can be computed with a Monte-Carlo procedure.

We thus focus on the following one-dimensional example:

$$
\partial_{t} u(t, x)+\frac{1}{2} \partial_{x, x}^{2} u(t, x)+|x|^{1 / 16}-|x-1|^{1 / 16}=0, \quad(t, x) \in[0,1) \times \mathbb{R},
$$

with the null boundary condition at time $T=1$. The second member counts two singularities in zero and one so that $u$ belongs to $C^{1+\alpha / 2,2+\alpha}([0, T] \times \mathbb{R}), \alpha=1 / 16$. On the opposite side, the solution $u$ has large "pockets" of regularity away from the singular points.

In light of Theorem 3.2, we thus expect the algorithm to behave poorly around the points zero and one and to be closer to the true solution away from them.

The solution, at time 0, may be expressed by the Feynman-Kac formula as

$$
u(0, x)=\mathbb{E}\left[\left|x+U^{1 / 2} Z\right|^{1 / 16}-\left|x+U^{1 / 2} Z-1\right|^{1 / 16}\right],
$$

where $U$ follows a uniform distribution on $(0,1)$ and is independent of $Z$ that is a standard Gaussian random variable. This permits us to compute a reference value with a Monte-Carlo method for different values of $x$. We plot below the reference values associated to $10^{6}$ simulations for $x$ varying in $[-5,5]$ with step .01 and then for $x$ around the first singularity, that is, for $x$ varying in $[-.5, .5]$ with step .005 . In each case, the empirical standard deviation is less than $10^{-1}$ so that the underlying error is at most of order $10^{-4}$.
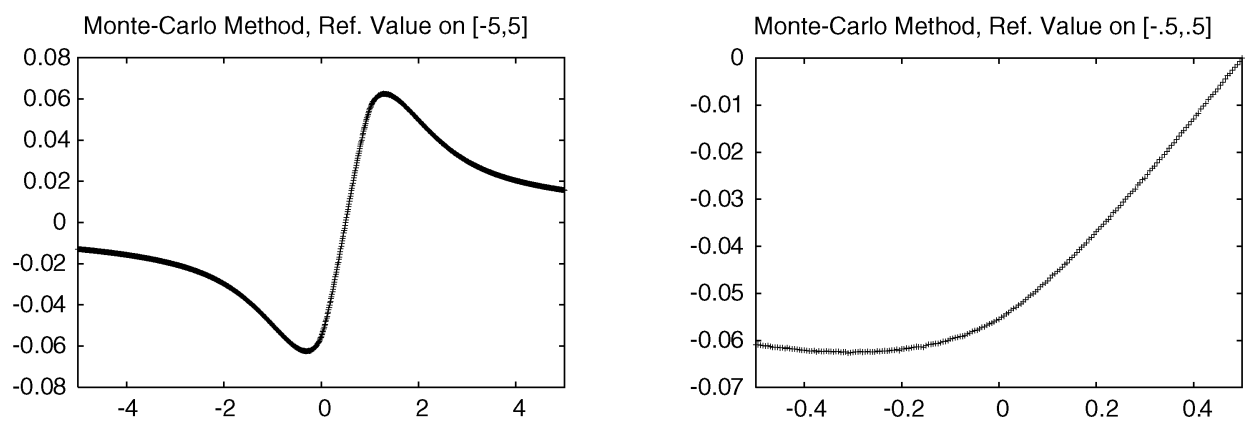

We plot below the absolute error on $[-.5, .5]$ between the reference value and the outcomes of our algorithm for the following choices: on the first row, $h=.01, \delta=$ .01 and $M=2,5,100$ and on the second row, $h=.001, \delta=.005$ and $M=2,5,100$. The Cartesian grid is truncated at the level $|x|=5$. 

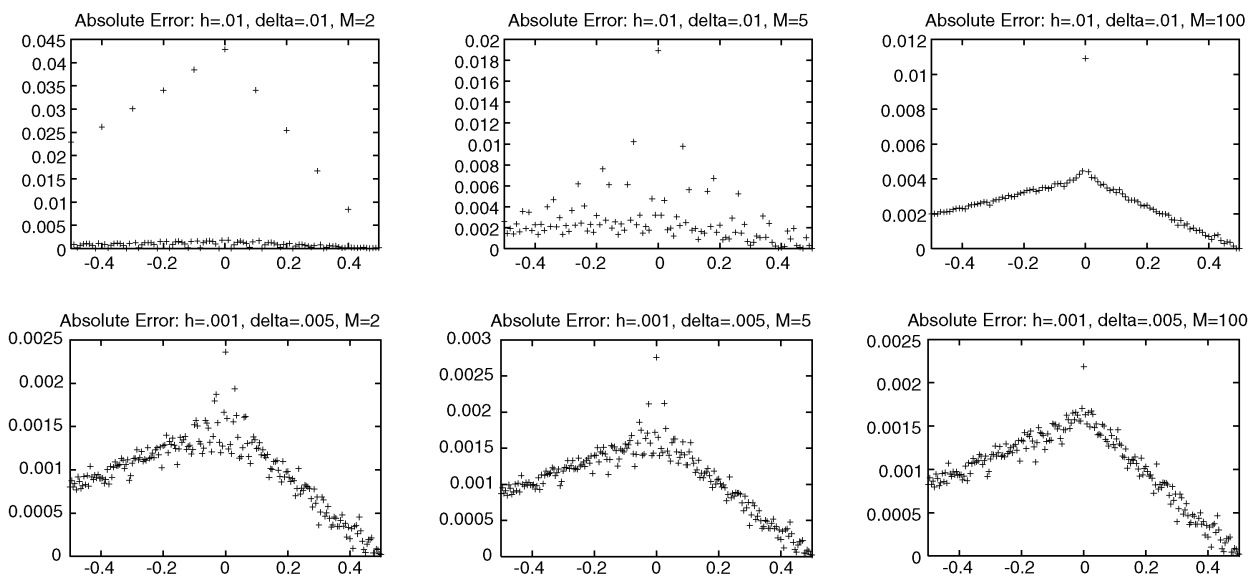

We will comment first on what happens in the case $h=.01$ and $\delta=.01$. Each graph exhibits jumps, especially around the singular point zero. The number of jumps as well as their magnitudes decrease with the size $M$ of the underlying quantization. The maximal error is close to .04 for $M=2$, to .02 for $M=5$ and to .01 for $M=100$. Moreover, for each value of $M$, the adequation is less and less satisfactory as it gets closer to the singular point zero and the maximal error is attained at $x=0$.

We provide here a possible explanation for all these observations: we feel that the algorithm, and more specifically the quantization procedure, is sensitive to the underlying local regularity of the coefficients. Indeed, it seems that each isolated singularity of the coefficients is propagated on a small neighborhood and induces several local jumps or "absurd" values. Conversely, we can reasonably think that the local error diminishes on "pockets" of smoothness. In the end, there might be a competition between the two phenomena, and the combination of both might vary with the number $M$ of quantization points: this might explain why quantization has an influence in the low regular setting. Of course, we have no proof for this interpretation and the reader may find it questionable: this point is to be investigated in further works.

Now we turn to the second case $h=.001$ and $\delta=.005$. Generally speaking, the results are more satisfactory than in the first case, as expected in light of Theorem 3.2 . For each value of $M$, the maximal error is between .002 and .003 , and the differences between them are of the same order as the error induced by the MonteCarlo method. Here is our interpretation: since $h$, which induces the dominant term in the global error, is now very small, jumps are now quite small and quantization doesn't seem to be as influent as in the former case. Anyhow, the fitting is still better away from the singular points: this seems to confirm the influence of the local smoothness of the coefficients and of the true solution.

\section{Proof, First step. Discrete FBSDEs}

To establish Theorem 3.2, we first express $\bar{u}_{\delta}$ through a discrete version of the FBSDE (E). Indeed, for each $k \in \llbracket 0, N)$, we can interpret the combination of the transition $\mathcal{T}\left(t_{k}, \cdot\right)$ and of the first order interpolation procedure as a Markovian kernel (see Lemma 5.1 and (5.2) ). In other words, we can build up, in Subsection 
5.2. a Markov process $\left(X_{t_{k}}\right)_{k \in \llbracket 0, N \rrbracket}$ such that $\left(\bar{u}_{\delta}\left(t_{k}, X_{t_{k}}\right)\right)_{k \in \llbracket 0, N \rrbracket}$ satisfies a discrete Feynman-Kac formula (see Proposition 5.2). Following the general theory for BSDEs (see [26]), we can associate, through the martingale representation theorem, a backward SDE to this Feynman-Kac formula (see (5.8)). In Proposition 5.3. we show that the representation term is mainly given by $\left(\bar{v}_{\delta}\left(t_{k}, X_{t_{k}}\right)\right)_{k \in \llbracket 0, N \rrbracket}$. The BSDE representation of $\bar{u}_{\delta}$ provides a maximum principle for Algorithm 3.1 and thus an $L^{\infty}$ bound for $\bar{u}_{\delta}$ (see Proposition (5.4) as well as an $L^{2}$ bound for $\bar{v}_{\delta}$ with respect to the density of the process $X$ (see Proposition 5.5), both bounds being independent of the discretization parameters. As a by-product, we prove that $\bar{v}_{\delta}$ is bounded, in $L^{\infty}$, by $h^{-1 / 2}$ up to a multiplicative constant.

About constants. In the following, we keep the same notation $C, C_{\eta}, c_{\eta}$ (or $\left.C^{\prime}, C_{\eta}^{\prime}, c_{\eta}^{\prime}\right)$ for all finite, non-negative constants which appear in our computations: they may depend on known parameters in (A), on $T$, but not on any of the discretization parameters. The index $\eta$ in the previous notation refers to the numbering of the proposition, lemma, theorem, etc., where the constant appears.

Conditions on parameters. The statements of the following propositions and lemmas hold for $h$ small enough.

5.1. Projection mappings. For a given point $x \in \mathbb{R}^{d}$, we want to individuate the cell it belongs to in order to determine the functions $\left(\phi_{z}\right)_{z \in \mathcal{C}_{\infty}}$ involved in the interpolation procedure. It is rather obvious that at most $2^{d}$ of them are concerned.

We first define the so-called projection to the lowest neighbor: $\forall x \in \mathbb{R}^{d}, \Pi^{(0)}(x)$ $=\left(\delta\left\lfloor\delta^{-1} x_{1}\right\rfloor, \cdots, \delta\left\lfloor\delta^{-1} x_{d}\right\rfloor\right)$. Following an arbitrary numbering, we denote by $\left.\Pi^{(i)}(x), i \in \llbracket 1,2^{d}\right)$, the remaining projection mappings to the vertices of the cell $x$ belongs to.

For $d=2, \Pi^{(0)}, \ldots, \Pi^{(3)}$ can be represented as in Figure 1 below.

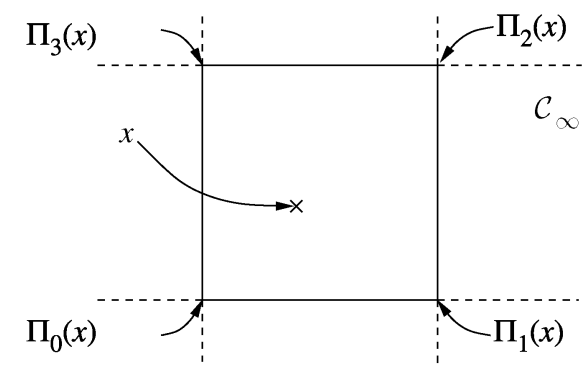

FiguRE 1. Projection mappings, $d=2$

With these notations, for a function $\psi: \mathcal{C}_{\infty} \rightarrow \mathbb{R}$, one also has

$$
\forall x \in \mathbb{R}^{d}, \psi_{\delta}(x)=\sum_{i=0}^{2^{d}-1} \phi_{\Pi^{(i)}(x)}(x) \psi\left(\Pi^{(i)}(x)\right) .
$$

As explained in Subsection 3.1, to analyze the convergence of Algorithm 3.1, we take advantage of the probabilistic interpretation of the piecewise linear interpolation. Indeed, for $x \in \mathbb{R}^{d}$, the family of non-negative weights $\left(\phi_{\Pi^{(i)}(x)}(x)\right)_{\left.i \in \llbracket 0,2^{d}\right)}$ 
defines a probability measure on the finite set $\left.\llbracket 0,2^{d}\right)$. We make use in the sequel of the associated cumulative distribution function

$$
\forall x \in \mathbb{R}^{d}, \alpha^{(0)}(x)=0, \forall i \in \llbracket 1,2^{d} \rrbracket, \alpha^{(i)}(x)=\sum_{j=0}^{i-1} \phi_{\Pi^{(j)}(x)}(x) .
$$

5.2. Discrete version of $(U, V, W)$. The key to prove Theorem 3.2 is to associate to Algorithm 3.1 a discrete version of the FBSDE (E). Assume for example that $(X, Y, Z)$ denotes a possible candidate to mimic $(U, V, W)$ but along Algorithm 3.1 . In order to have a discrete Feynman-Kac formula, we need to define the forward process $\left(X_{t_{k}}\right)_{k \in \llbracket 0, N \rrbracket}$ as a Markov chain with continuous state space. Since the transitions in the algorithm are defined on the grid, we need to specify the transition for $\left(X_{t_{k}}\right)_{k \in \llbracket 0, N)}$ according to its spatial position at the current discretization time. The most natural way to proceed consists in randomly choosing, at time $t_{k}$, one of the $2^{d}$ possible transitions associated to the cell that $X_{t_{k}}$ belongs to. This is done by simulating, independently of the Brownian motion, a discrete random variable with weights $\left(\phi_{k}^{(i)} \equiv \phi_{\Pi^{(i)}\left(X_{t_{k}}\right)}\left(X_{t_{k}}\right)\right)_{\left.i \in \llbracket 0,2^{d}\right)}$.

Extension of the probability space. Let $\left(U_{\ell}\right)_{\ell \in \mathbb{N}^{*}}$ be a sequence of independent identically distributed random variables, independent of the $\sigma$-field $\bigvee_{t \geq 0} \mathcal{F}_{t}^{B}$, such that $U_{1} \sim \mathcal{U}([0,1])$. We set, for $t \geq 0, \mathcal{F}_{t} \equiv \mathcal{F}_{t}^{B} \vee \mathcal{F}_{t}^{U}$, with $\mathcal{F}_{t}^{U} \equiv \sigma\left(U_{1}, \ldots, U_{k+1}\right)$ with $k$ such that $t_{k}<t \leq t_{k+1}\left(\mathcal{F}_{0}^{\bar{U}} \equiv\{\emptyset, \Omega\}\right)$. In particular, the $\sigma$-field $\mathcal{F}_{t+}^{U}$ differs from $\mathcal{F}_{t}^{U}$ for $t \in\left\{\left(t_{k}\right)_{k \in \llbracket 0, N)}\right\}$. For simplicity, we set $\forall k \in \llbracket 0, N \rrbracket, \mathbb{E}_{k}[.] \equiv \mathbb{E}\left[. \mid \mathcal{F}_{t_{k}}\right]$.

The following lemma (whose proof is left to the reader) provides the connection between the variables $\left(U_{\ell}\right)_{\ell \in \mathbb{N}^{*}}$ and the projection mappings.

Lemma 5.1. Conditionally to the $\sigma$-field $\left.\mathcal{F}_{t_{k}}, k \in \llbracket 0, N\right), U_{k+1}$ and $\sigma\left(B_{s}, s \leq t_{k+1}\right)$ are independent and for every $\mathbb{R}^{d}$-valued and $\mathcal{F}_{t_{k}}$-measurable random variable $\xi$ :

$$
\left.\forall i \in \llbracket 0,2^{d}\right), \mathbb{E}_{k}\left[\mathbf{I}_{U_{k+1} \in\left[\alpha^{(i)}(\xi), \alpha^{(i+1)}(\xi)[\right.}\right]=\phi_{\Pi^{(i)}(\xi)}(\xi) .
$$

Discrete representation processes. Algorithm 3.1 and Lemma 5.1 motivate, for an initial condition $x_{0} \in \mathbb{R}^{d}$, the following definition for the approximating triple $(X, Y, Z)$. Set $X_{0} \equiv x_{0}$ and

$$
\begin{aligned}
X_{t_{k+1}} & \equiv \sum_{i=0}^{2^{d}-1} \mathrm{I}_{U^{k+1} \in\left[\alpha^{(i)}\left(X_{t_{k}}\right), \alpha^{(i+1)}\left(X_{t_{k}}\right)[\right.}\left(\Pi^{(i)}\left(X_{t_{k}}\right)+\mathcal{T}\left(t_{k}, \Pi^{(i)}\left(X_{t_{k}}\right)\right)\right) \\
& \left.\equiv \sum_{i=0}^{2^{d}-1} \chi_{k+1}^{(i)} X_{t_{k+1}}^{(i)} \text { for } k \in \llbracket 0, N\right) \\
\left(Y_{t_{k}}, Z_{t_{k}}\right) & \equiv\left(\bar{u}_{\delta}\left(t_{k}, X_{t_{k}}\right), \bar{v}_{\delta}\left(t_{k}, X_{t_{k}}\right)\right) \text { for } k \in \llbracket 0, N \rrbracket .
\end{aligned}
$$

Note carefully that $X_{t_{k+1}}^{(i)}$ does not stand for the $i^{\text {th }}$ component of a vector of $\mathbb{R}^{d}$. It is associated to the initial position $\Pi^{(i)}\left(X_{t_{k}}\right)$ and to the transition $\mathcal{T}\left(t_{k}, \Pi^{(i)}\left(X_{t_{k}}\right)\right)$. Referring to the notations introduced after Algorithm $3.1, \mathcal{T}\left(t_{k}, \Pi^{(i)}\left(X_{t_{k}}\right)\right)$ writes in an obvious manner $\mathcal{T}\left(t_{k}, \Pi^{(i)}\left(X_{t_{k}}\right)\right) \equiv b_{k}^{(i)} h+\sigma_{k}^{(i)} \gamma^{*} g\left(\Delta B^{k}\right)$. 
Backward equation. From the above definition we derive the following:

Proposition 5.2 (Discrete Feynman Kac formula). For all $k \in \llbracket 0, N)$,

$$
\begin{aligned}
Y_{t_{k}} & =\mathbb{E}_{k}\left[H\left(X_{t_{N}}\right)\right. \\
& \left.+h \sum_{j=k}^{N-1} \sum_{i=0}^{2^{d}-1} \phi_{j}^{(i)} f\left(\Pi^{(i)}\left(X_{t_{j}}\right), \bar{u}\left(t_{j+1}, \Pi^{(i)}\left(X_{t_{j}}\right)\right), \bar{v}\left(t_{j}, \Pi^{(i)}\left(X_{t_{j}}\right)\right)\right)\right] \\
& \equiv \mathbb{E}_{k}\left[H\left(X_{t_{N}}\right)+h \sum_{j=k}^{N-1} \sum_{i=0}^{2^{d}-1} \phi_{j}^{(i)} f_{j}^{(i)}\right] \equiv \mathbb{E}_{k}\left[H\left(X_{t_{N}}\right)+h \sum_{j=k}^{N-1} \bar{f}_{\delta}\left(t_{j}, X_{t_{j}}\right)\right] .
\end{aligned}
$$

Proof. Write first, for $k \in \llbracket 0, N$ ) (we specify over the symbols "=" the references employed for the computations)

$$
\begin{aligned}
& \mathbb{E}_{k}\left[Y_{t_{k+1}}\right] \stackrel{\sqrt{5.21}}{=} \mathbb{E}_{k}\left[\bar{u}_{\delta}\left(t_{k+1}, X_{t_{k+1}}\right) \stackrel{\text { (3.3) }}{=} \sum_{z \in \mathcal{C}_{\infty}} \phi u\left(t_{k+1}, z\right) \mathbb{E}_{k}\left[\phi_{z}\left(X_{t_{k+1}}\right)\right]\right. \\
& \stackrel{\text { 5.2. }}{=} \text { Le. } \stackrel{5.1}{=} \sum_{z \in \mathcal{C}_{\infty}} \phi u\left(t_{k+1}, z\right) \sum_{i=0}^{2^{d}-1} \mathbb{E}_{k}\left[\chi_{k+1}^{(i)}\right] \mathbb{E}_{k}\left[\phi_{z}\left(X_{t_{k+1}}^{(i)}\right)\right] \\
& \stackrel{\text { Le.5.1] }}{=} \sum_{z \in \mathcal{C}_{\infty}} \phi u\left(t_{k+1}, z\right) \sum_{i=0}^{2^{d}-1} \phi_{k}^{(i)} \mathbb{E}_{k}\left[\phi_{z}\left(X_{t_{k+1}}^{(i)}\right)\right] .
\end{aligned}
$$

From Algorithm 3.1, one also gets

$$
\begin{aligned}
& Y_{t_{k}} \stackrel{(5.2)}{=} \bar{u}_{\delta}\left(t_{k}, X_{t_{k}}\right) \stackrel{(5.1)}{=} \sum_{i=0}^{2^{d}-1} \phi_{k}^{(i)} \emptyset u\left(t_{k}, \Pi^{(i)}\left(X_{t_{k}}\right)\right) \\
& \stackrel{\text { Al. }}{\underline{3.1}} \sum_{i=0}^{2^{d}-1} \phi_{k}^{(i)}\left\{\mathbb{E}_{k}\left[\bar{u}_{\delta}\left(t_{k+1}, X_{t_{k+1}}^{(i)}\right)\right]+h f_{k}^{(i)}\right\}
\end{aligned}
$$

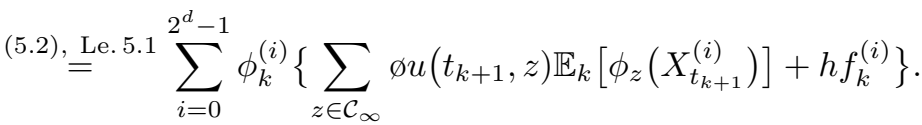

Equations (5.3) and (5.4) yield $\mathbb{E}_{k}\left[Y_{t_{k+1}}\right]+h \sum_{i=0}^{2^{d}-1} \phi_{k}^{(i)} f_{k}^{(i)}=Y_{t_{k}}$. The proof of the proposition follows by induction.

5.3. Associated a priori estimates. From (5.2), we derive $\forall k \in \llbracket 0, N)$

$$
Y_{t_{k+1}}=\sum_{i=0}^{2^{d}-1} \chi_{k+1}^{(i)} \bar{u}_{\delta}\left(t_{k+1}, X_{t_{k+1}}^{(i)}\right) .
$$

For $k \in \llbracket 0, N)$ and $\left.i \in \llbracket 0,2^{d}\right)$, the random variable $\bar{u}_{\delta}\left(t_{k+1}, X_{t_{k+1}}^{(i)}\right)$ is

$$
\mathcal{F}_{t_{k}} \vee \sigma\left(B_{t}-B_{t_{k}}, t_{k} \leq t \leq t_{k+1}\right)
$$

measurable. Thanks to the Martingale Representation Theorem (see e.g. Theorem III.4.33 in Jacod and Shiryaev [14]), there exists a process $\left(\varnothing Z_{t}^{(i)}\right)_{t_{k} \leq t \leq t_{k+1}}$ with 
values in $\mathbb{R}^{d}$, progressively measurable with respect to the previous filtration and with finite moment of order two, such that:

$$
\bar{u}_{\delta}\left(t_{k+1}, X_{t_{k+1}}^{(i)}\right)=\mathbb{E}_{k}\left[\bar{u}_{\delta}\left(t_{k+1}, X_{t_{k+1}}^{(i)}\right)\right]+\int_{t_{k}}^{t_{k+1}} \bar{Z}_{s}^{(i)} d B_{s}
$$

so that $Y_{t_{k+1}}=\sum_{i=0}^{2^{d}-1} \chi_{k+1}^{(i)}\left\{\mathbb{E}_{k}\left[\bar{u}_{\delta}\left(t_{k+1}, X_{t_{k+1}}^{(i)}\right)\right]+\int_{t_{k}}^{t_{k+1}} \bar{Z}_{s}^{(i)} d B_{s}\right\}$. Define for $t \in$ $\left[t_{k}, t_{k+1}\right]$,

$$
\bar{Z}_{t}=\sum_{i=0}^{2^{d}-1} \chi_{k+1}^{(i)} \bar{Z}_{t}^{(i)} .
$$

Now rewrite

$$
\begin{aligned}
Y_{t_{k+1}} & =\sum_{i=0}^{2^{d}-1} \phi_{k}^{(i)} \mathbb{E}_{k}\left[\bar{u}_{\delta}\left(t_{k+1}, X_{t_{k+1}}^{(i)}\right)\right]+\int_{t_{k}}^{t_{k+1}} \varnothing Z_{s} d B_{s}+\Delta R_{k+1}(1) \\
& \stackrel{\text { Le. [5.1 }}{=} \mathbb{E}_{k}\left[Y_{t_{k+1}}\right]+\int_{t_{k}}^{t_{k+1}} \varnothing Z_{s} d B_{s}+\Delta R_{k+1}(1), \\
\Delta R_{k+1}(1) & \equiv \sum_{i=0}^{2^{d}-1}\left(\chi_{k+1}^{(i)}-\phi_{k}^{(i)}\right) \mathbb{E}_{k}\left[\bar{u}_{\delta}\left(t_{k+1}, X_{t_{k+1}}^{(i)}\right)\right] .
\end{aligned}
$$

Note from Lemma 5.1 that $\mathbb{E}_{k}\left[\Delta R_{k+1}(1)\right]=0$. Eventually use Proposition 5.2 to obtain:

$$
\begin{aligned}
& Y_{t_{k+1}}=Y_{t_{k}}-h \varnothing f_{\delta}\left(t_{k}, X_{t_{k}}\right)+\int_{t_{k}}^{t_{k+1}} \phi Z_{s} d B_{s}+\Delta R_{k+1}(1), \\
& Y_{t_{N}}+h \sum_{k=1}^{N} \varnothing f_{\delta}\left(t_{k-1}, X_{t_{k-1}}\right)=Y_{0}+\int_{0}^{T} \bar{Z}_{s} d B_{s}+\sum_{k=1}^{N} \Delta R_{k}(1) .
\end{aligned}
$$

This allows us to apply the BSDE machinery already used in 9], provided the following a priori estimates.

Proposition 5.3. For $k \in \llbracket 1, N \rrbracket, h Z_{t_{k-1}}=\mathbb{E}_{k-1}\left[\int_{t_{k-1}}^{t_{k}} \bar{Z}_{s} d s\right] \gamma$ and for $\left.i \in \llbracket 0,2^{d}\right)$, $h \varnothing v\left(t_{k-1}, \Pi^{(i)}\left(X_{t_{k-1}}\right)\right)=\mathbb{E}_{k-1}\left[\int_{t_{k-1}}^{t_{k}} \varnothing Z_{s}^{(i)} d s\right] \gamma$.

Proposition 5.4. $\exists C_{5.4} \geq 0$ s.t. $\sup _{(k, x) \in \llbracket 0, N \rrbracket \times \mathbb{R}^{d}}\left|\bar{u}_{\delta}\left(t_{k}, x\right)\right|^{2} \leq C_{5.4 .}$.

Proposition 5.5. $\exists C \overline{5.5} \geq 0$ s.t. $\mathbb{E}\left[\int_{0}^{T}\left|\bar{Z}_{s}\right|^{2} d s\right]+h \sum_{k=0}^{N-1} \mathbb{E}\left[|\bar{v}|_{\delta}^{2}\left(t_{k}, X_{t_{k}}\right)+\left|Z_{t_{k}}\right|^{2}\right]$ $+h \sup _{(k, x) \in \llbracket 0, N \rrbracket \times \mathbb{R}^{d}}\left|\bar{v}_{\delta}\left(t_{k}, x\right)\right|^{2} \leq C_{5.5}$.

Proof of Proposition 5.3. From (5.8), write for a given $k \in \llbracket 0, N)$ :

$$
\begin{aligned}
& Y_{t_{k+1}}+h \varnothing f_{\delta}\left(t_{k}, X_{t_{k}}\right)=Y_{t_{k}}+\int_{t_{k}}^{t_{k+1}} \bar{Z}_{s} d B_{s}+\Delta R_{k+1}(1),
\end{aligned}
$$

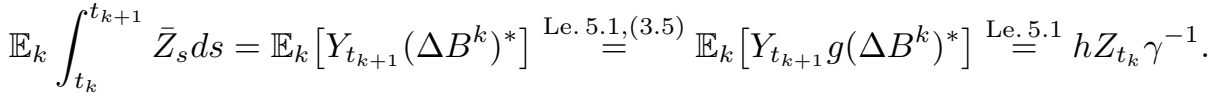

Similar arguments and (5.5) yield the second statement of the proposition. 
Proof of Proposition 5.4. We apply the basic strategy of the BSDE theory using a discrete version of Itô's formula; see Shiryaev [28], Chapter VII, Subsection 9 or Lemma 6.8 in 9 . We get:

$$
\left|Y_{T}\right|^{2}=\left|Y_{0}\right|^{2}+2 \sum_{k=1}^{N}\left\langle Y_{t_{k}}-Y_{t_{k-1}}, Y_{t_{k-1}}\right\rangle+\sum_{k=1}^{N}\left|Y_{t_{k}}-Y_{t_{k-1}}\right|^{2},
$$

with $Y_{t_{k}}-Y_{t_{k-1}}=-h \varnothing f_{\delta}\left(t_{k-1}, X_{t_{k-1}}\right)+\int_{t_{k-1}}^{t_{k}} \bar{Z}_{s} d B_{s}+\Delta R_{k}(1)$ (cf. (5.8)). From (5.7), $\mathbb{E}_{k-1}\left[\Delta R_{k}(1)\right]=0$. Similarly,

$$
\begin{aligned}
& \mathbb{E}_{k-1}\left[\int_{t_{k-1}}^{t_{k}} \bar{Z}_{s} d B_{s}\right] \stackrel{\sqrt[(5.6)]{=}}{=} \sum_{i=0}^{2^{d}-1} \mathbb{E}_{k-1}\left[\chi_{k}^{(i)} \int_{t_{k-1}}^{t_{k}} \bar{Z}_{s}^{(i)} d B_{s}\right]
\end{aligned}
$$

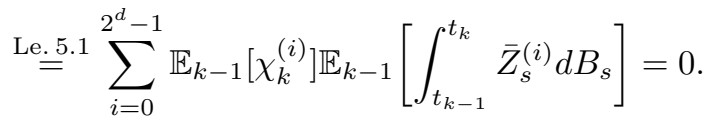

Hence,

$$
\begin{aligned}
\mathbb{E}\left|Y_{T}\right|^{2}= & \left|Y_{0}\right|^{2}+2 h \sum_{k=1}^{N} \mathbb{E}\left\langle-\varnothing f_{\delta}\left(t_{k-1}, X_{t_{k-1}}\right), Y_{t_{k-1}}\right\rangle \\
& +\sum_{k=1}^{N} \mathbb{E}\left[\left\{h \varnothing f_{\delta}\left(t_{k-1}, X_{t_{k-1}}\right)-\int_{t_{k-1}}^{t_{k}} \bar{Z}_{s} d B_{s}-\Delta R_{k}(1)\right\}^{2}\right] .
\end{aligned}
$$

As above, the expectations of the double products involving $\varnothing f_{\delta}\left(t_{k-1}, X_{t_{k-1}}\right)$ and $\int_{t_{k-1}}^{t_{k}} \bar{Z}_{s} d B_{s}$ on the one hand and $\varnothing f_{\delta}\left(t_{k-1}, X_{t_{k-1}}\right)$ and $\Delta R_{k}(1)$ on the other hand vanish. Note finally that

$$
\begin{aligned}
& \mathbb{E}_{k-1}\left[\left(\int_{t_{k-1}}^{t_{k}} \bar{Z}_{s} d B_{s}\right) \Delta R_{k}(1)\right] \\
& \stackrel{\sqrt[5.6]{=}}{=} \sum_{i=0}^{2^{d}-1} \mathbb{E}_{k-1}\left[\left(\int_{t_{k-1}}^{t_{k}} \bar{Z}_{s}^{(i)} d B_{s}\right)\left(\chi_{k}^{(i)} \Delta R_{k}(1)\right)\right] \\
& \stackrel{\text { Le. }}{=} \sum_{i=0}^{2^{d}-1} \mathbb{E}_{k-1}\left[\left(\int_{t_{k-1}}^{t_{k}} \bar{Z}_{s}^{(i)} d B_{s}\right)\right] \mathbb{E}_{k-1}\left[\chi_{k}^{(i)} \Delta R_{k}(1)\right]=0 .
\end{aligned}
$$

Plug (5.9) and (5.11) into (5.10):

$$
\begin{aligned}
& \mathbb{E}\left|Y_{T}\right|^{2}=\left|Y_{0}\right|^{2}+2 h \sum_{k=1}^{N} \mathbb{E}\left\langle-\varnothing f_{\delta}\left(t_{k-1}, X_{t_{k-1}}\right), Y_{t_{k-1}}\right\rangle \\
& +h^{2} \sum_{k=1}^{N} \mathbb{E}\left[\varnothing f_{\delta}^{2}\left(t_{k-1}, X_{t_{k-1}}\right)\right]+\mathbb{E} \int_{0}^{T}\left|\bar{Z}_{s}\right|^{2} d s+\sum_{k=1}^{N} \mathbb{E}\left[\Delta R_{k}^{2}(1)\right] .
\end{aligned}
$$


From Assumption (A) and (3.2), there exists a constant $C$ such that:

$$
\begin{aligned}
\left|Y_{0}\right|^{2}+\mathbb{E} \int_{0}^{T}\left|\bar{Z}_{s}\right|^{2} d s & \leq \mathbb{E}\left|Y_{T}\right|^{2}+C h \sum_{k=1}^{N} \mathbb{E}\left[| Y _ { t _ { k - 1 } } | \left(1+\left|\bar{u}_{\delta}\left(t_{k}, \cdot\right)\right|_{\infty}\right.\right. \\
& \left.\left.+\sum_{i=0}^{2^{d}-1} \phi_{k-1}^{(i)}\left|\varnothing v\left(t_{k-1}, \Pi_{i}\left(X_{t_{k-1}}\right)\right)\right|\right)\right] .
\end{aligned}
$$

From Young's inequality and from Jensen's inequality (applied to interpolation weights), we derive for every $\eta>0$ :

$$
\begin{aligned}
& \left|Y_{0}\right|^{2}+\mathbb{E} \int_{0}^{T}\left|\bar{Z}_{s}\right|^{2} d s \leq \mathbb{E}\left|Y_{T}\right|^{2}+C \eta^{-1} h \sum_{k=1}^{N} \mathbb{E}\left[\left|Y_{t_{k-1}}\right|^{2}\right] \\
& +C \eta h \sum_{k=1}^{N} \mathbb{E}\left[1+\left|\bar{u}_{\delta}\left(t_{k}, \cdot\right)\right|_{\infty}^{2}+\sum_{i=0}^{2^{d}-1} \phi_{k-1}^{(i)}|\varnothing v|^{2}\left(t_{k-1}, \Pi_{i}\left(X_{t_{k-1}}\right)\right)\right] .
\end{aligned}
$$

Set $Q \equiv h \sum_{k=1}^{N} \sum_{i=0}^{2^{d}-1} \mathbb{E}\left[\phi_{k-1}^{(i)}|\varnothing v|^{2}\left(t_{k-1}, \Pi_{i}\left(X_{t_{k-1}}\right)\right)\right]$. Owing to Proposition 5.3 .

$$
\begin{aligned}
Q & =h^{-1} \sum_{k=1}^{N} \sum_{i=0}^{2^{d}-1} \mathbb{E}\left[\phi_{k-1}^{(i)}\left|\mathbb{E}_{k-1}\left[\int_{t_{k-1}}^{t_{k}} \phi Z_{s}^{(i)} d s\right] \gamma\right|^{2}\right] \\
& \leq C \sum_{k=1}^{N} \sum_{i=0}^{2^{d}-1} \mathbb{E}\left[\phi_{k-1}^{(i)} \mathbb{E}_{k-1}\left[\int_{t_{k-1}}^{t_{k}}\left|\varnothing Z_{s}^{(i)}\right|^{2} d s\right]\right] .
\end{aligned}
$$

Now write

$$
Q \stackrel{\text { Le. [5.1 }}{\leq} C \sum_{k=1}^{N} \sum_{i=0}^{2^{d}-1} \mathbb{E}\left[\chi_{k}^{(i)} \int_{t_{k-1}}^{t_{k}}\left|\varnothing Z_{s}^{(i)}\right|^{2} d s\right] \stackrel{(5.6)}{=} C \mathbb{E}\left[\int_{0}^{T}\left|\varnothing Z_{s}\right|^{2} d s\right] .
$$

From (5.15) and (5.14), we derive that for $\eta$ and $h$ small enough

$$
\left|Y_{0}\right|^{2}+\frac{1}{2} \mathbb{E}\left[\int_{0}^{T}\left|\varnothing Z_{s}\right|^{2} d s\right] \leq \mathbb{E}\left|Y_{T}\right|^{2}+C+C h \sum_{k=1}^{N}\left|\bar{u}_{\delta}\left(t_{k}, \cdot\right)\right|_{\infty}^{2} .
$$

Recall that $\left|Y_{T}\right| \leq|H|_{\infty}$. Thus $\left|\bar{u}_{\delta}(0, \cdot)\right|_{\infty}^{2} \leq C+C h \sum_{k=1}^{N}\left|\bar{u}_{\delta}\left(t_{k}, \cdot\right)\right|_{\infty}^{2}$. As usual in BSDE theory, we could establish in a similar way that for every $j \in \llbracket 1, N)$ : $\left|\bar{u}_{\delta}\left(t_{j}, \cdot\right)\right|_{\infty}^{2} \leq C+C h \sum_{k=j+1}^{N}\left|\bar{u}_{\delta}\left(t_{k}, \cdot\right)\right|_{\infty}^{2}$. A discrete version of Gronwall's Lemma yields the result.

Proof of Proposition 5.5. The $L^{2}$-estimate of $\varnothing Z$ follows from Proposition 5.4 and (5.16). Then, the $L^{2}$-estimate of $Z$ follows from the $L^{2}$-estimate of $\varnothing Z$ and Proposition 5.3. The $L^{2}$-estimate of $|\bar{v}|_{\delta}$ then comes from the earlier definition of $Q$. Finally, as a consequence of Proposition 5.4 and the definition of $\bar{v}_{\delta}$, see Algorithm 3.1. we deduce the estimate of the supremum norm of $\bar{v}_{\delta}$. 


\section{Proof, Second step: Stability Properties}

This section focuses on the second step of the proof of Theorem 3.2. Our strategy follows from the decoupling argument (or Four Step Scheme) used by Ma, Protter and Yong [20] to establish uniqueness of the solutions to some FBSDEs. Indeed, because of the smoothness properties of the true solution $u$ (see Theorem 2.1), we can express $\left(u\left(t_{k}, X_{t_{k}}\right)\right)_{k \in \llbracket 0, N \rrbracket}$ as the solution of a new BSDE (see (6.7)). The whole point is to compare this new BSDE to the one satisfied by $\left(Y_{t_{k}}\right)_{k \in \llbracket 0, N \rrbracket}$ : this amounts to applying to our specific setting general stability properties for BSDEs. The minutely detailed computations are postponed to the end of the section (see Lemmas 6.3 and 6.4). The final result is stated below (see Theorem 6.1). Theorem 3.2 then follows from an obvious combination of Theorem 6.1 and Gronwall's Lemma.

To express $\left(u\left(t_{k}, X_{t_{k}}\right)\right)_{k \in \llbracket 0, N \rrbracket}$ as the solution of a BSDE, we first introduce a time continuous extension of $X$ (see (6.2) $)$ and then develop $\left(u\left(t, X_{t}\right)\right)_{0 \leq t \leq T}$ by Itô's formula. The dynamics of $X$ between $t_{k}$ and $\left.t_{k+1}, k \in \llbracket 0, N\right)$, is chosen to be Gaussian, so that $X$ jumps at time $t_{k}$. We investigate in Lemma 6.2 the sizes of these jumps.

6.1. Stability theorem. Applying the usual FBSDE machinery, we establish in Subsection 6.2.

Theorem 6.1. There exists a constant $C_{6.1}>0$ such that:

$$
\begin{aligned}
& \left|\left(\bar{u}_{\delta}-u\right)(0, x)\right|^{2}+C_{6.1]}^{-1} h \sum_{k=1}^{N} \mathbb{E}\left[\left(|\bar{v}-v|^{2}\right)_{\delta}\left(t_{k-1}, X_{t_{k-1}}\right)\right] \\
& \leq C_{\overline{6.1}[}\left[\mathcal{E}^{2}(\text { global })+h \sum_{k=1}^{N}\left|\left(\bar{u}_{\delta}-u\right)\left(t_{k}, \cdot\right)\right|_{\infty}^{2}\right] .
\end{aligned}
$$

We then derive Theorem 3.2 from Theorem 6.1 and Gronwall's Lemma (up to a modification of the initial condition).

\subsection{Proof of Theorem 6.1.}

Starting point: Time continuous processes. For the proof, we need to extend the definition of $X$ to the whole set $[0, T]$. Put for all $k \in \llbracket 0, N)$ and $t \in\left(t_{k}, t_{k+1}\right)$ :

$$
\begin{aligned}
X_{t} & \equiv \sum_{i=0}^{2^{d}-1} \chi_{k+1}^{(i)}\left[\Pi^{(i)}\left(X_{t_{k}}\right)+b_{k}^{(i)}\left(t-t_{k}\right)+\sigma_{k}^{(i)} \gamma^{*}\left(B_{t}-B_{t_{k}}\right)\right] \\
& \equiv \sum_{i=0}^{2^{d}-1} \chi_{k+1}^{(i)} X_{t}^{(i)} .
\end{aligned}
$$

Note that $X_{t_{k}+}^{(i)}=\Pi^{(i)}\left(X_{t_{k}}\right)$. Hence, the extended process $\left(X_{t}\right)_{0 \leq t \leq T}$ is discontinuous at times $\left(t_{k}\right)_{k \in \llbracket 1, N)}$, both at $t_{k}-$ and $t_{k}+$ (of course, it is also discontinuous at times $0+$ and $T-)$. At a given time $\left.t_{k}, k \in \llbracket 1, N\right)$, the size of the jumps performed by the process depends, on the right, on the spatial projection error, and, on the left, on the quantization error. The first one is bounded by $\delta$ and is of mean zero. The second one is easily controlled by the distortion; cf. (3.4). More precisely, for 
all $k \in \llbracket 0, N)$

$$
X_{t_{k+1}}-X_{t_{k+1}-}=\sum_{i=0}^{2^{d}-1}\left[\chi_{k+1}^{(i)} \sigma_{k}^{(i)} \gamma^{*}\left[g\left(\Delta B^{k}\right)-\Delta B^{k}\right]\right] .
$$

Moreover, one easily obtains the following

Lemma 6.2. There exists $C_{6.2} \geq 0$ s.t. for every $k \in \llbracket 0, N-1 \rrbracket$ :

$$
\left.\forall i \in \llbracket 0,2^{d}\right), \forall t \in\left(t_{k}, t_{k+1}\right), \mathbb{E}_{k}\left[\left|X_{t}^{(i)}-X_{t_{k}+}^{(i)}\right|^{2}\right] \leq C_{\overline{6.2}} h .
$$

Referring to the structure of the $\operatorname{PDE}(\mathcal{E})$, we set

$$
\begin{aligned}
& \forall t \in[0, T], \varnothing V_{t} \equiv u\left(t, X_{t}\right), \bar{W}_{t} \equiv \nabla_{x} u\left(t, X_{t}\right) \sigma\left(X_{t}, \bar{V}_{t}\right), \\
& \left.\forall t \notin\left\{\left(t_{k}\right)_{k \in \llbracket 0, N \rrbracket}\right\}, i \in \llbracket 0,2^{d}\right),\left\{\begin{array}{c}
\bar{V}_{t}^{(i)} \equiv u\left(t, X_{t}^{(i)}\right), \\
\bar{W}_{t}^{(i)} \equiv \nabla_{x} u\left(t, X_{t}^{(i)}\right) \sigma\left(X_{t}^{(i)}, \bar{V}_{t}^{(i)}\right) \\
\equiv \nabla_{x} u\left(t, X_{t}^{(i)}\right) \bar{\sigma}_{t}^{(i)} .
\end{array}\right.
\end{aligned}
$$

Note, moreover, that the martingale part of $\left(\bar{V}_{t}\right)_{0 \leq t \leq T}$ is driven, for $t \in\left(t_{k}, t_{k+1}\right)$, $k \in \llbracket 0, N)$, by:

$$
W_{t} \gamma^{*} \equiv \sum_{i=0}^{2^{d}-1}\left[\chi_{k+1}^{(i)} \nabla_{x} u\left(t, X_{t}^{(i)}\right) \sigma_{k}^{(i)} \gamma^{*}\right] \equiv \sum_{i=0}^{2^{d}-1}\left[\chi_{k+1}^{(i)} W_{t}^{(i)} \gamma^{*}\right]
$$

From Theorem 2.1 and Lemma 6.2, we derive the following a priori estimates for $\left.\bar{V}^{(i)}, \bar{W}^{(i)}, i \in \llbracket 0,2^{d}\right)$. For all $\left.k \in \llbracket 0, N\right)$ and $s \in\left(t_{k}, t_{k+1}\right)$,

$$
\mathbb{E}_{k}\left[\left|\bar{V}_{s}^{(i)}-\bar{V}_{t_{k}+}^{(i)}\right|^{2}+\left|\bar{W}_{s}^{(i)}-\bar{W}_{t_{k}+}^{(i)}\right|^{2}\right] \leq C h .
$$

Step One: Itô's formula for $\bar{V}$. Applying Itô's formula to $\left(u\left(t, X_{t}^{(i)}\right)\right)_{t_{k}<t<t_{k+1}}$, for all $\left.i \in \llbracket 0,2^{d}\right)$ and $\left.k \in \llbracket 0, N\right)$, and using the $\operatorname{PDE}(\mathcal{E})$, we obtain:

$$
\begin{gathered}
\bar{V}_{t_{k+1}}-\bar{V}_{t_{k}}=\bar{V}_{t_{k+1}}-\bar{V}_{t_{k+1}-}+\bar{V}_{t_{k}+}-\bar{V}_{t_{k}} \\
+\sum_{i=0}^{2^{d}-1}\left\{\chi _ { k + 1 } ^ { ( i ) } \left[\int _ { t _ { k } } ^ { t _ { k + 1 } } \left[F\left(s, X_{s}^{(i)}, X_{t_{k}+}^{(i)}, \bar{u}\left(t_{i+1}, X_{t_{k}+}^{(i)}\right), \sigma_{k}^{(i)} \gamma^{*} \gamma\left(\sigma_{k}^{(i)}\right)^{*}\right)\right.\right.\right. \\
\left.-F\left(s, X_{s}^{(i)}, X_{s}^{(i)}, \bar{V}_{s}^{(i)}, \bar{\sigma}_{s}^{(i)}\left(\bar{\sigma}_{s}^{(i)}\right)^{*}\right)\right] d s \\
\left.\left.\quad-\int_{t_{k}}^{t_{k+1}} f\left(X_{s}^{(i)}, \bar{V}_{s}^{(i)}, \bar{W}_{s}^{(i)}\right) d s\right]\right\}+\int_{t_{k}}^{t_{k+1}} W_{s} \gamma^{*} d B_{s},
\end{gathered}
$$

with $F\left(s, x, x^{\prime}, y, A\right)=(1 / 2) \operatorname{tr}\left(A H_{u}(s, x)\right)+\nabla_{x} u(s, x) b\left(x^{\prime}, y\right), s \in[0, T], x, x^{\prime} \in \mathbb{R}^{d}$, $y \in \mathbb{R}$ and $A \in \mathbb{R}^{d \times d}$.

Step Two: Difference of the processes. The strategy is well-known: make the difference between $\bar{V}$ and $Y$ and apply the usual BSDE machinery to estimate the 
difference. From Proposition 5.2 and (5.8), we claim:

$$
\begin{aligned}
& \bar{V}_{t_{k+1}}-Y_{t_{k+1}}-\left[\bar{V}_{t_{k}}-Y_{t_{k}}\right] \\
& =\left\{\bar{V}_{t_{k+1}}-\bar{V}_{t_{k+1}-}+\Delta R_{k+1}(3)\right\}+\left\{\bar{V}_{t_{k}+}-\bar{V}_{t_{k}}\right\} \\
& +\left\{\sum _ { i = 0 } ^ { 2 ^ { d } - 1 } \chi _ { k + 1 } ^ { ( i ) } \left[\int _ { t _ { k } } ^ { t _ { k + 1 } } \left[F\left(s, X_{s}^{(i)}, X_{t_{k}+}^{(i)}, \bar{u}\left(t_{i+1}, X_{t_{k}+}^{(i)}\right), \sigma_{k}^{(i)}\left(\sigma_{k}^{(i)}\right)^{*}\right)\right.\right.\right. \\
& \left.-F\left(s, X_{s}^{(i)}, X_{s}^{(i)}, \bar{V}_{s}^{(i)}, \bar{\sigma}_{s}^{(i)}\left(\bar{\sigma}_{s}^{(i)}\right)^{*}\right)\right] \\
& \left.\left.\left.-f\left(X_{s}^{(i)}, \bar{V}_{s}^{(i)}, \bar{W}_{s}^{(i)}\right)+f_{k}^{(i)}\right] d s\right]\right\} \\
& +\left\{\int_{t_{k}}^{t_{k+1}}\left[W_{s} \gamma^{*}-\bar{Z}_{s}\right] d B_{s}+\Delta R_{k+1}(2)\right\} \\
& \equiv \Delta E_{k+1}(1)+\Delta E_{k+1}(2)+\Delta E_{k+1}(3)+\Delta E_{k+1}(4),
\end{aligned}
$$

denoting

$$
\begin{aligned}
& \Delta R_{k+1}(2) \equiv h \sum_{i=0}^{2^{d}-1}\left[\left(\phi_{k}^{(i)}-\chi_{k+1}^{(i)}\right) f_{k}^{(i)}\right]-\Delta R_{k+1}(1), \\
& \Delta R_{k+1}(3) \equiv \sum_{i=0}^{2^{d}-1} \chi_{k+1}^{(i)} \int_{t_{k}}^{t_{k+1}} \frac{1}{2} \operatorname{tr}\left(H_{u}\left(s, X_{s}^{(i)}\right) \sigma_{k}^{(i)}\left(\gamma^{*} \gamma-I_{d}\right)\left(\sigma_{k}^{(i)}\right)^{*}\right) d s .
\end{aligned}
$$

The discrete Itô formula yields:

$$
\begin{aligned}
\mathbb{E}\left|\bar{V}_{T}-Y_{T}\right|^{2}= & \left|\bar{V}_{0}-Y_{0}\right|^{2}+2 \mathbb{E} \sum_{k=1}^{N}\left[\left[\bar{V}_{t_{k-1}}-Y_{t_{k-1}}\right]\left(\sum_{\ell=1}^{4} \Delta E_{k}(\ell)\right)\right] \\
& +\mathbb{E} \sum_{k=1}^{N}\left[\left(\sum_{\ell=1}^{4} \Delta E_{k}(\ell)\right)^{2}\right] .
\end{aligned}
$$

From the above expression, we get:

$$
\begin{aligned}
\left|\bar{V}_{0}-Y_{0}\right|^{2}+\sum_{k=1}^{N} \mathbb{E}\left[\left(\Delta E_{k}(1)+\Delta E_{k}(2)+\Delta E_{k}(4)\right)^{2}\right] \\
=\mathbb{E}\left|\bar{V}_{T}-Y_{T}\right|^{2}-2 \mathbb{E} \sum_{k=1}^{N}\left[\left[\bar{V}_{t_{k-1}}-Y_{t_{k-1}}\right]\left(\sum_{\ell=1}^{4} \Delta E_{k}(\ell)\right)\right] \\
\quad-\sum_{k=1}^{N} \mathbb{E}\left[\left(\Delta E_{k}(3)\right)^{2}\right]-2 \sum_{k=1}^{N} \mathbb{E}\left[\Delta E_{k}(3)\left(\Delta E_{k}(1)+\Delta E_{k}(2)+\Delta E_{k}(4)\right)\right] .
\end{aligned}
$$

The inequality $2 a b \leq 2 a^{2}+(1 / 2) b^{2}$ yields

$$
\begin{aligned}
\mid \bar{V}_{0} & -\left.Y_{0}\right|^{2}+\frac{1}{2} \sum_{k=1}^{N} \mathbb{E}\left[\left(\Delta E_{k}(1)+\Delta E_{k}(2)+\Delta E_{k}(4)\right)^{2}\right] \\
\leq & \mathbb{E}\left|\bar{V}_{T}-Y_{T}\right|^{2} \\
& -2 \mathbb{E} \sum_{k=1}^{N}\left[\left[\bar{V}_{t_{k-1}}-Y_{t_{k-1}}\right]\left(\sum_{\ell=1}^{4} \Delta E_{k}(\ell)\right)\right]+\sum_{k=1}^{N} \mathbb{E}\left[\left(\Delta E_{k}(3)\right)^{2}\right] .
\end{aligned}
$$


Put:

$$
\begin{aligned}
D(1) & \equiv-2 \mathbb{E} \sum_{k=1}^{N}\left[\left[\bar{V}_{t_{k-1}}-Y_{t_{k-1}}\right]\left(\sum_{\ell=1}^{4} \Delta E_{k}(\ell)\right)\right], \\
D(2) & \equiv \sum_{k=1}^{N} \mathbb{E}\left[\left(\Delta E_{k}(3)\right)^{2}\right], \\
D(3) & \equiv \sum_{k=1}^{N} \mathbb{E}\left[\left(\Delta E_{k}(1)+\Delta E_{k}(2)+\Delta E_{k}(4)\right)^{2}\right] .
\end{aligned}
$$

Step Three: Standard BSDE techniques. Admit for the moment that

Lemma 6.3. There exists a constant $C[6.3$ such that for $\eta \in] 0,1]$ :

$$
\begin{aligned}
|D(1)|+D(2) \leq & \left.C \mathcal{E}^{2} \text { (global }\right)+C h \eta^{-1} \sum_{k=1}^{N}\left|\left(\bar{u}_{\delta}-u\right)\left(t_{k}, .\right)\right|_{\infty}^{2} \\
& +h(\eta+h) \sum_{k=1}^{N} \mathbb{E}\left[\left(|\bar{v}-v|^{2}\right)_{\delta}\left(t_{k-1}, X_{t_{k-1}}\right)\right] .
\end{aligned}
$$

Lemma 6.4. There exists a constant $C \overline{6.4}>0$ such that:

$$
\begin{aligned}
D(3) \geq & G_{[6.4}^{-1} h \sum_{k=1}^{N} \mathbb{E}\left[\left(|\bar{v}-v|^{2}\right)_{\delta}\left(t_{k-1}, X_{t_{k-1}}\right)\right]-C \overline{6.4} \mathcal{E}^{2} \text { (global) } \\
& -C_{6.4} h \sum_{k=1}^{N}\left|\left(\bar{u}_{\delta}-u\right)\left(t_{k-1}, .\right)\right|_{\infty}^{2} .
\end{aligned}
$$

To conclude the proof of Theorem 6.1 note that $Y_{T}=\bar{V}_{T}$. Hence, for $\eta$ small enough one obtains inequality (6.1) from (6.11), (6.12), and Lemmas 6.3 and 6.4 This completes, up to the proofs of Lemmas 6.3 and 6.4 the proof of Theorem 6.1 .

Proof of Lemma 6.3, D(1). From Theorem 2.1, Lemma 5.1] and Taylor's formula applied to $\bar{V}_{t_{k}}-\bar{V}_{t_{k}-}=\sum_{i=0}^{2^{d}-1}\left[\chi_{k}^{(i)}\left(u\left(t_{k}, X_{t_{k}}^{(i)}\right)-u\left(t_{k}, X_{t_{k}-}^{(i)}\right)\right)\right]$, we derive

$$
\begin{aligned}
& \mathbb{E}_{k-1}\left[\Delta E_{k}(1)\right] \\
& =\sum_{i=0}^{2^{d}-1} \phi_{k-1}^{(i)}\left\{\mathbb{E}_{k-1}\left[\nabla_{x} u\left(t_{k}, X_{t_{k}}^{(i)}\right)\left(X_{t_{k}}^{(i)}-X_{t_{k}-}^{(i)}\right)\right]\right. \\
& +\mathbb{E}_{k-1}\left[\int _ { t _ { k - 1 } } ^ { t _ { k } } d s \int _ { 0 } ^ { 1 } d \lambda ( 1 - \lambda ) \left(\operatorname{tr}\left(H_{u}\left(s, X_{s}^{(i)}\right) \sigma_{k-1}^{(i)}\left(\gamma^{*} \gamma-I_{d}\right)\left(\sigma_{k-1}^{(i)}\right)^{*}\right)\right.\right. \\
& -h^{-1} \operatorname{tr}\left(H_{u}\left(t_{k}, X_{t_{k}}^{(i), \lambda}\right) \sigma_{k-1}^{(i)} \gamma^{*}\left[\left(g\left(\Delta B^{k-1}\right)-\Delta B^{k-1}\right)\right]\right. \\
& \left.\left.\left.\left.\times\left[\left(g\left(\Delta B^{k-1}\right)-\Delta B^{k-1}\right)\right]^{*} \gamma\left(\sigma_{k-1}^{(i)}\right)^{*}\right)\right)\right]\right\}
\end{aligned}
$$

with $X_{t_{k}}^{(i), \lambda} \equiv X_{t_{k}}^{(i)}+\lambda\left(X_{t_{k}-}^{(i)}-X_{t_{k}}^{(i)}\right)$. Exploiting (3.5), we get

$$
\begin{aligned}
& \mathbb{E}_{k-1}\left[\nabla_{x} u\left(t_{k}, X_{t_{k}}^{(i)}\right)\left(X_{t_{k}}^{(i)}-X_{t_{k}-}^{(i)}\right)\right] \\
& =\mathbb{E}_{k-1}\left[\nabla_{x} u\left(t_{k}, X_{t_{k}}^{(i)}\right) \sigma_{k-1}^{(i)} \gamma^{*} \mathbb{E}\left[g\left(\Delta B^{k-1}\right)-\Delta B^{k-1} \mid g\left(\Delta B^{k-1}\right)\right]\right]=0 .
\end{aligned}
$$


Thus with obvious notations

$$
\mathbb{E}_{k-1}\left[\Delta E_{k}(1)\right] \equiv \sum_{i=0}^{2^{d}-1} \phi_{k-1}^{(i)} \int_{t_{k-1}}^{t_{k}} d s \int_{0}^{1} d \lambda(1-\lambda) \mathbb{E}_{k-1}\left[I_{k-1}^{(i)}(s, \lambda)\right] .
$$

Noting that $h^{-1} \mathbb{E}_{k-1}\left[\left(g\left(\Delta B^{k-1}\right)-\Delta B^{k-1}\right)\left(g\left(\Delta B^{k-1}\right)-\Delta B^{k-1}\right)^{*}\right]=I_{d}-\left(\gamma \gamma^{*}\right)^{-1}$, we derive

$$
\begin{aligned}
& \mathbb{E}_{k-1}\left[I_{k-1}^{(i)}(s, \lambda)\right] \\
& =\mathbb{E}_{k-1}\left[\operatorname{tr}\left(\left[H_{u}\left(s, X_{s}^{(i)}\right)-H_{u}\left(t_{k-1}, X_{t_{k-1}+}^{(i)}\right)\right] \sigma_{k-1}^{(i)}\left(\gamma^{*} \gamma-I_{d}\right)\left(\sigma_{k-1}^{(i)}\right)^{*}\right)\right] \\
& -\mathbb{E}_{k-1}\left[h ^ { - 1 } \operatorname { t r } \left(\left[H_{u}\left(t_{k}, X_{t_{k}}^{(i), \lambda}\right)-H_{u}\left(t_{k-1}, X_{t_{k-1}+}^{(i)}\right)\right]\right.\right. \\
& \left.\left.\quad \times \sigma_{k-1}^{(i)} \gamma^{*}\left[\left(g\left(\Delta B^{k-1}\right)-\Delta B^{k-1}\right)\right]\left[\left(g\left(\Delta B^{k-1}\right)-\Delta B^{k-1}\right)\right]^{*} \gamma\left(\sigma_{k-1}^{(i)}\right)^{*}\right)\right] .
\end{aligned}
$$

From (3.4), note first that $\left|\gamma \gamma^{*}-I_{d}\right| \leq C M^{-2 / d}$. The key idea is now to use the $\alpha$-Hölder continuity of the Hessian matrix of $u$, cf. Theorem 2.1, combined with the fact that for $\beta \in[2, d+2)$, the square Gaussian quantization is still rate optimal in $L^{\beta}(\mathbb{P})$, cf. [11, that is, $\left(\mathbb{E}\left[\left|g\left(\Delta B^{k-1}\right)-\Delta B^{k-1}\right|^{\beta}\right]\right)^{\beta^{-1}} \leq C h^{1 / 2} M^{-1 / d}$. Hence, putting $\zeta>1$ s.t. $\zeta^{-1}+(\beta / 2)^{-1}=1$, the Hölder inequality yields:

$$
\begin{aligned}
& \left|\mathbb{E}_{k-1}\left[I_{k-1}^{(i)}(s, \lambda)\right]\right| \\
& \leq C \mathbb{E}_{k-1}\left[\left(h^{\alpha / 2}+\sup _{t_{k-1}<s<t_{k}}\left|X_{s}^{(i)}-X_{t_{k-1}+}^{(i)}\right|^{\alpha}+\left|X_{t_{k}-}^{(i)}-X_{t_{k}}^{(i)}\right|^{\alpha}\right)^{\zeta}\right]^{\zeta^{-1}} \\
& \quad \times\left[M^{-2 / d}+h^{-1} \mathbb{E}_{k-1}\left[\left|\Delta B^{k-1}-g\left(\Delta B^{k-1}\right)\right|^{\beta}\right]^{2 \beta^{-1}}\right] \leq C h^{\alpha / 2} M^{-2 / d} .
\end{aligned}
$$

Plug the above control into (6.14) to obtain $\left|\mathbb{E}_{k-1}\left[\Delta E_{k}(1)\right]\right| \leq C h^{1+\alpha / 2} M^{-2 / d}$.

Turn to $\Delta E_{k}(2)$. Apply the Taylor formula to the function $u$ :

$$
\begin{aligned}
& \mathbb{E}_{k-1}\left[\Delta E_{k}(2)\right]=\mathbb{E}_{k-1}\left[\sum_{i=0}^{2^{d}-1} \chi_{k}^{(i)}\left(u\left(t_{k-1}, X_{t_{k-1}+}^{(i)}\right)-u\left(t_{k-1}, X_{t_{k-1}}\right)\right)\right] \\
& =\sum_{i=0}^{2^{d}-1} \phi_{k-1}^{(i)}\left[u\left(t_{k-1}, X_{t_{k-1}+}^{(i)}\right)-u\left(t_{k-1}, X_{t_{k-1}}\right)\right] \\
& =\sum_{i=0}^{2^{d}-1} \phi_{k-1}^{(i)}\left[\nabla_{x} u\left(t_{k-1}, X_{t_{k-1}}\right)\left(X_{t_{k-1}+}^{(i)}-X_{t_{k-1}}\right)\right. \\
& \quad+\int_{0}^{1} d \lambda(1-\lambda) \operatorname{tr}\left[H_{u}\left(t_{k-1}, X_{t_{k-1}}+\lambda\left(X_{t_{k-1}+}^{(i)}-X_{t_{k-1}}\right)\right)\right. \\
& \left.\left.\quad \times\left(X_{t_{k-1}+}^{(i)}-X_{t_{k-1}}\right)\left(X_{t_{k-1}+}^{(i)}-X_{t_{k-1}}\right)^{*}\right]\right] \\
& \equiv \sum_{i=0}^{2^{d}-1} \phi_{k-1}^{(i)}\left[\Delta E_{k}^{(i)}(2,1)+\Delta E_{k}^{(i)}(2,2)\right] .
\end{aligned}
$$

Since the shape functions $\left(\phi_{z}\right)_{z \in \mathcal{C}_{\infty}}$ are chosen to interpolate exactly the polynomials of order less than one, we have $\sum_{i=0}^{2^{d}-1} \phi_{k-1}^{(i)} \Delta E_{k}^{(i)}(2,1)=0$. Moreover, the regularity properties of $u$ yield $\left|\sum_{i=0}^{2^{d}-1} \phi_{k-1}^{(i)} \Delta E_{k}^{(i)}(2,2)\right| \leq C \delta^{2}$, so that $\left|\mathbb{E}_{k-1}\left[\Delta E_{k}(2)\right]\right|$ $\leq C h \mathcal{E}$ (space). 
Due to (5.7), (5.9) and (6.9), the contribution of $\Delta E(4)$ in $D(1)$ is null.

Now note from Theorem 2.1 (boundedness of the first and second order derivatives) that $\Delta E(3)$ may be seen as a "Hölder/Lipschitz" difference. Additionally, using the above controls for the conditional expectations of $\Delta E(1)$ and $\Delta E(2)$, we derive that there exists a constant $C$, such that:

$$
\begin{aligned}
|D(1)| \leq & C \mathbb{E} \sum_{k=1}^{N}\left\{| \overline { V } _ { t _ { k - 1 } } - Y _ { t _ { k - 1 } } | \left[h \mathcal{E}(\text { global })+\sum_{i=0}^{2^{d}-1}\left[\phi_{k-1}^{(i)}\right.\right.\right. \\
& \times\left(\int_{t_{k-1}}^{t_{k}}\left[\left|X_{s}^{(i)}-X_{t_{k-1}+}^{(i)}\right|^{\alpha}+\left|\bar{V}_{s}^{(i)}-\bar{u}\left(t_{k}, X_{t_{k-1}+}^{(i)}\right)\right|\right] d s\right. \\
& \left.\left.\left.\left.+\int_{t_{k-1}}^{t_{k}}\left[\left|\bar{W}_{s}^{(i)}-\varnothing v\left(t_{k-1}, X_{t_{k-1}+}^{(i)}\right)\right|\right] d s\right)\right]\right]\right\} .
\end{aligned}
$$

Recall that $\bar{V}_{s}^{(i)}=u\left(s, X_{s}^{(i)}\right), \varnothing W_{s}^{(i)}=v\left(s, X_{s}^{(i)}\right)$. From Theorem 2.1 (Hölder regularity of $u$ in $t$ ), (6.6) (regularity of $\bar{V}$ and $\bar{W}$ ) and Lemma 6.2 (control of the increments of $X$ ), we then deduce:

$$
\begin{aligned}
|D(1)| \leq & C \mathbb{E} \sum_{k=1}^{N}\left[| \overline { V } _ { t _ { k - 1 } } - Y _ { t _ { k - 1 } } | \left(h \mathcal{E}(\text { global })+h^{1+\alpha / 2}\right.\right. \\
& \left.\left.+h\left\{\left|\left(\bar{u}_{\delta}-u\right)\left(t_{k}, \cdot\right)\right|_{\infty}+|\varnothing v-v|_{\delta}\left(t_{k-1}, X_{t_{k-1}}\right)\right\}\right)\right]
\end{aligned}
$$

Since $h^{1+\alpha / 2}=h \mathcal{E}$ (time), we derive, from Young's inequality, the required control for $D(1)$.

Proof for $D(2)$. Recall that $D(2)=\mathbb{E} \sum_{k=1}^{N}\left[\left(\Delta E_{k}(3)\right)^{2}\right]$. Again, the terms $\Delta E_{k}(3)$ are "Hölder/Lipschitz" differences, so that the strategy used to obtain (6.15) applies. This provides the required bound for $D(2)$.

\subsubsection{Proof of Lemma 6.4. Recall that}

$$
D(3)=\sum_{k=1}^{N} \mathbb{E}\left[\left(\Delta E_{k}(1)+\Delta E_{k}(2)+\int_{t_{k-1}}^{t_{k}}\left[W_{s} \gamma^{*}-\bar{Z}_{s}\right] d B_{s}+\Delta R_{k}(2)\right)^{2}\right]
$$

Now, sticking to the notations introduced for the proof of Lemma 6.3 (see (6.13) $)$, Taylor's formula applied to $\Delta E_{k} S(1)$ yields: 


$$
\begin{aligned}
& \int_{t_{k-1}}^{t_{k}}\left[W_{s} \gamma^{*}-\varnothing Z_{s}\right] d B_{s}+\Delta E_{k}(1) \\
& =\left\{\sum_{i=0}^{2^{d}-1} \chi_{k}^{(i)}\left(v\left(t_{k-1}, X_{t_{k-1}+}^{(i)}\right) \gamma^{*} g\left(\Delta B^{k-1}\right)-\int_{t_{k-1}}^{t_{k}} \varnothing Z_{s}^{(i)} d B_{s}\right)\right\} \\
& +\left\{\sum_{i=0}^{2^{d}-1} \chi_{k}^{(i)}\left[\nabla_{x} u\left(t_{k}, X_{t_{k}}^{(i)}\right) \sigma_{k-1}^{(i)}-v\left(t_{k-1}, X_{t_{k-1}+}^{(i)}\right)\right] \gamma^{*}\left(g\left(\Delta B^{k-1}\right)-\Delta B^{k-1}\right)\right. \\
& -\sum_{i=0}^{2^{d}-1} \chi_{k}^{(i)} \int_{0}^{1} d \lambda(1-\lambda) \operatorname{tr}\left[H_{u}\left(t_{k}, X_{t_{k}}^{(i), \lambda}\right) \sigma_{k-1}^{(i)} \gamma^{*}\left(g\left(\Delta B^{k-1}\right)-\Delta B^{k-1}\right)\right. \\
& \left.\quad \times\left(g\left(\Delta B^{k-1}\right)-\Delta B^{k-1}\right)^{*} \gamma\left(\sigma_{k-1}^{(i)}\right)^{*}\right] \\
& \left.+\sum_{i=0}^{2^{d}-1} \chi_{k}^{(i)} \int_{t_{k-1}}^{t_{k}}\left[W_{s}^{(i)}-v\left(t_{k-1}, X_{t_{k-1}+}^{(i)}\right)\right] \gamma^{*} d B_{s}+\Delta R_{k}(3)\right\} \\
& \equiv A_{k}(1)+A_{k}(2) .
\end{aligned}
$$

From Theorem 2.1, (6.9) and standard estimates for the increments of $X$, we deduce $\sum_{k=1}^{N} \mathbb{E}\left[\left(A_{k}(2)\right)^{2}\right] \leq C h$. Thus, $D(3) \geq(1 / 2) \sum_{k=1}^{N} \mathbb{E}\left[\left(A_{k}(1)+\Delta R_{k}(2)+\Delta E_{k}(2)\right)^{2}\right]$ $-C h$. From (5.11), (6.9) and Lemma 5.1, we already know that the covariance between the stochastic integral (respectively the quantized Brownian increment) and $\Delta R_{k}(2)$ vanishes. Moreover,

$$
\begin{aligned}
& \mathbb{E}_{k-1}\left[A_{k}(1) \Delta E_{k}(2)\right] \\
& =\sum_{i=0}^{2^{d}-1} \mathbb{E}_{k-1}\left[\chi _ { k } ^ { ( i ) } \left(v\left(t_{k-1}, X_{t_{k-1}+}^{(i)}\right) \gamma^{*} g\left(\Delta B^{k-1}\right)\right.\right. \\
& \left.\left.-\int_{t_{k-1}}^{t_{k}} \bar{Z}_{s}^{(i)} d B_{s}\right)\left(u\left(t_{k-1}, X_{t_{k-1}+}^{(i)}\right)-u\left(t_{k-1}, X_{t_{k-1}}\right)\right)\right]=0 .
\end{aligned}
$$

We finally obtain

$$
\begin{aligned}
D(3) \geq & \frac{1}{2} \sum_{k=1}^{N} \sum_{i=0}^{2^{d}-1} \mathbb{E}\left[\phi _ { k - 1 } ^ { ( i ) } \mathbb { E } _ { k - 1 } \left[\left(v\left(t_{k-1}, X_{t_{k-1}+}^{(i)}\right) \gamma^{*} g\left(\Delta B^{k-1}\right)\right.\right.\right. \\
& \left.\left.\left.-\int_{t_{k-1}}^{t_{k}} \varnothing Z_{s}^{(i)} d B_{s}\right)^{2}\right]\right]-C h .
\end{aligned}
$$

To investigate (6.16), we follow (6.2) and put $X_{t_{k}}^{(i)}=\Pi^{(i)}\left(X_{t_{k-1}}\right)+b_{k-1}^{(i)} h+\sigma_{k-1}^{(i)} \gamma^{*}$ $\times g\left(\Delta B^{k-1}\right.$ ) (in (6.2),$X_{t}^{(i)}$ is just defined for $t_{k-1}<t<t_{k}$ ). A new application of the Taylor formula at order one to $u\left(t_{k}, X_{t_{k}}^{(i)}\right)$ with respect to the initial value $\left(t_{k-1}, X_{t_{k-1}+}^{(i)}\right)$ yields, for all $\left.i \in \llbracket 0,2^{d}\right)$ and $k \in \llbracket 1, N \rrbracket, u\left(t_{k}, X_{t_{k}}^{(i)}\right)=$ $u\left(t_{k-1}, X_{t_{k-1}+}^{(i)}\right)+\nabla_{x} u\left(t_{k-1}, X_{t_{k-1}+}^{(i)}\right)\left(X_{t_{k}}^{(i)}-X_{t_{k-1}+}^{(i)}\right)+O(h)$, so that

$$
\begin{gathered}
\mathbb{E}_{k-1}\left[\left(u\left(t_{k}, X_{t_{k}}^{(i)}\right)-\mathbb{E}_{k-1}\left[u\left(t_{k}, X_{t_{k}}^{(i)}\right)\right]-v\left(t_{k-1}, X_{t_{k-1}+}^{(i)}\right) \gamma^{*} g\left(\Delta B^{k-1}\right)\right)^{2}\right] \\
\leq C\left(h^{2}+h\left|\left(\bar{u}_{\delta}-u\right)\left(t_{k-1}, .\right)\right|_{\infty}^{2}\right) .
\end{gathered}
$$


Hence, denoting by $\mathbb{V}_{k-1}$ the conditional variance with respect to $\mathcal{F}_{t_{k-1}},(\underline{6.16}$ ) and (5.5) give

$$
\begin{aligned}
& D(3) \geq \frac{1}{4} \sum_{k=1}^{N} \sum_{i=0}^{2^{d}-1} \mathbb{E}\left[\phi_{k-1}^{(i)} \mathbb{V}_{k-1}\left[\left(u-\bar{u}_{\delta}\right)\left(t_{k}, X_{t_{k}}^{(i)}\right)\right]\right] \\
&-C\left(h+h \sum_{k=1}^{N}\left|\left(\bar{u}_{\delta}-u\right)\left(t_{k-1}, .\right)\right|_{\infty}^{2}\right) .
\end{aligned}
$$

Since $v\left(t_{k-1}, X_{t_{k-1}+}^{(i)}\right)=h^{-1} \mathbb{E}_{k-1}\left[v\left(t_{k-1}, X_{t_{k-1}+}^{(i)}\right) \gamma^{*} g\left(\Delta B^{k-1}\right) g\left(\Delta B^{k-1}\right)^{*} \gamma\right]$, (6.17) and the Cauchy-Schwarz inequality yield

$$
\begin{aligned}
& \left|\mathbb{E}_{k-1}\left[h^{-1} u\left(t_{k}, X_{t_{k}}^{(i)}\right) g\left(\Delta B^{k-1}\right)^{*} \gamma\right]-v\left(t_{k-1}, X_{t_{k-1}+}^{(i)}\right)\right| \\
& =h^{-1} \mid \mathbb{E}_{k-1}\left[\left(u\left(t_{k}, X_{t_{k}}^{(i)}\right)-\mathbb{E}_{k-1}\left[u\left(t_{k}, X_{t_{k}}^{(i)}\right)\right]\right.\right. \\
& \left.\left.\quad \quad-v\left(t_{k-1}, X_{t_{k-1}+}^{(i)}\right) \gamma^{*} g\left(\Delta B^{k-1}\right)\right) g\left(\Delta B^{k-1}\right)^{*} \gamma\right] \mid \\
& \leq C\left(h+\left|\left(\bar{u}_{\delta}-u\right)\left(t_{k-1}, .\right)\right|_{\infty}^{2}\right)^{1 / 2}
\end{aligned}
$$

so that

$$
\begin{aligned}
& h\left|(v-\bar{v})\left(t_{k-1}, X_{t_{k-1}+}^{(i)}\right)\right|^{2} \\
& \leq C h^{-1}\left|\mathbb{E}_{k-1}\left[\left(u-\bar{u}_{\delta}\right)\left(t_{k}, X_{t_{k}}^{(i)}\right) g\left(\Delta B^{k-1}\right)^{*} \gamma\right]\right|^{2}+C\left(h^{2}+h\left|\left(\bar{u}_{\delta}-u\right)\left(t_{k-1}, .\right)\right|_{\infty}^{2}\right) \\
& \leq C \mathbb{V}_{k-1}\left[\left(u-\bar{u}_{\delta}\right)\left(t_{k}, X_{t_{k}}^{(i)}\right)\right]+C\left(h^{2}+h\left|\left(\bar{u}_{\delta}-u\right)\left(t_{k-1}, .\right)\right|_{\infty}^{2}\right) .
\end{aligned}
$$

Due to (6.18), this completes the proof.

\section{Convergence When $b$ Depends on the Gradient}

As announced in Section 3, we now investigate the case where $b$ depends on $v$.

As already explained, when $b$ is bounded, we can apply Algorithm 3.1 to the fourtuple $(0, g, H, \sigma)$ (see the explanations after (3.6) for the meaning of this notation), with $g(x, u, v)=f(x, u, v)+v \sigma^{-1}(x, u) b(x, u, v)$. Indeed, although the coefficient $g$ does not satisfy Assumption (A), Theorem 3.2 still holds in this case. Let us review the main arguments: first, since $b$ is bounded, $g$ is of linear growth, so that all the bounds given in Section 5 are true in this new setting; second, we can plug the bound for the gradient of the true solution $u$ (see Theorem 2.1) in the regularity estimate (3.6) and treat the term $\Delta E_{k+1}(3)$ in (6.8) as a Lispchitz difference.

In the general frame, we are not able to establish the convergence of Algorithm 3.1 for the four-tuple $(b, f, \sigma, H)$, or for the four-tuple $(0, g, \sigma, H)$ either. The reason was already discussed in our previous paper [9]: one way or another, the analysis of Algorithm 3.1 leads us to investigate the regularity of the approximated gradient $\bar{v}$. This task is certainly far from being trivial.

Intermediate predictor. The common strategy consists in introducing an intermediate predictor for $v$, denoted by $\hat{v}$, for which the underlying estimates are easier to establish. In [9], $\left.\hat{v}\left(t_{k}, \cdot\right), k \in \llbracket 0, N\right)$, is given as a regularized version of $\bar{v}\left(t_{k+1}, \cdot\right)$. Our choice in the current paper is slightly different. For $k \in \llbracket 0, N)$ and $x \in \mathcal{C}_{\infty}$, set:

$$
\begin{aligned}
& \hat{v}\left(t_{k}, x\right) \equiv h^{-1} \mathbb{E}\left[\bar{u}_{\delta}\left(t_{k+1}, x+\mathcal{T}^{0}\left(t_{k}, x\right)\right) g\left(\Delta B^{k}\right)^{*}\right] \gamma \\
& \mathcal{T}^{0}\left(t_{k}, x\right) \equiv \Sigma\left(t_{k}, x\right) \gamma^{*} g\left(\Delta B^{k}\right) .
\end{aligned}
$$


Define the transition $\mathcal{T}\left(t_{k}, x\right) \equiv \beta\left(t_{k}, x\right) h+\Sigma\left(t_{k}, x\right) \gamma^{*} g\left(\Delta B^{k}\right)$ with $\beta\left(t_{k}, x\right) \equiv$ $b\left(x, \bar{u}\left(t_{k+1}, x\right), \hat{v}\left(t_{k}, x\right)\right), \Sigma\left(t_{k}, x\right) \equiv \sigma\left(x, \bar{u}\left(t_{k+1}, x\right)\right) \gamma^{*}$. The definitions of $\bar{u}\left(t_{k}, x\right)$ and $\bar{v}\left(t_{k}, x\right)$ then remain the same (according to the new choice for $\mathcal{T}\left(t_{k}, x\right)$ ).

The above definition (7.1) is in fact quite natural: when replacing $\bar{u}_{\delta}$ by the true solution $u$, the first order approximation of $h^{-1} \mathbb{E}\left[u\left(t_{k+1}, x+\mathcal{T}^{0}\left(t_{k}, x\right)\right) g\left(\Delta B^{k}\right)^{*}\right] \gamma$ remains $v\left(t_{k}, x\right)$ itself.

Shape functions. To investigate the convergence of the above algorithm, we also need to change the way we interpolate the approximated solution at the nodes of the grid. Indeed, for our analysis, we require $\bar{u}_{\delta}$ to be sufficiently smooth in $x$. A possible strategy consists in choosing a $B$-spline basis instead of a family of Lagrange kernels of order one; see e.g. the monograph of de Boor [6]. Namely, choose $\Phi$ in (3.1) as $\Phi(t)=2 / 3-t^{2}+|t|^{3} / 2$ for $|t| \leq 1, \Phi(t)=(1 / 6)(2-|t|)^{3}$ for $1 \leq|t| \leq 2$ and $\Phi(t)=0$ for $|t| \geq 2$. This $\Phi$ is nonnegative and, for all $x \in \mathbb{R}^{d}$, $\sum_{z \in \mathcal{C}_{\infty}} \phi_{z}(x)=1$ and $\sum_{z \in \mathcal{C}_{\infty}} z \phi_{z}(x)=x$, so that (3.2) holds. Of course, this new choice for $\Phi$ changes the number of "useful neighbors" in (5.1): there are now $4^{d}$ instead of $2^{d}$ neighbors to consider. Anyhow, the probabilistic interpretation of Lemma 5.1 still holds: we let the reader adapt the definitions of the functions $\Pi^{(i)}$, $\left.i \in \llbracket 0,4^{d}\right)$, in (5.1).

As expected, the approximated solution $\bar{u}_{\delta}$ then belongs to $\mathcal{C}^{2}$ with Lipschitz derivatives of order one and two. Moreover, the interpolation procedure associated to the spline basis being exact for polynomials of order less than one is of order two for smooth functions:

Lemma 7.1. Choose $\Phi$ as above in (3.1). Then, for a real $\delta>0$ and for a function $\psi \in \mathcal{C}^{2}\left(\mathbb{R}^{d}, \mathbb{R}\right)$ with bounded second order derivatives, the interpolated function $\psi_{\delta}$ given by (3.3) satisfies, for all $x \in \mathbb{R}^{d},\left|\psi_{\delta}(x)-\psi(x)\right| \leq C_{7.1}(\psi) \delta^{2}$, for a constant $C_{7.1}(\psi)$ only depending on the supremum norm of $H_{\psi}$.

Main result. Here is the main result of this section:

Theorem 7.2. Assume that $b$ depends on $v$. Then, taking into account the above modifications of Algorithm 3.1, there exist two constants $q_{7.2}>0$ and $C_{7.2}$, only depending on $T$ and on known parameters appearing in $(\mathbf{A})$, such that, for $\mathcal{E}$ (global) $<$ q7.2 (with $\mathcal{E}$ (global) as in Theorem [3.2) and for $\mathcal{B}$ (gradient) $\equiv 1+\delta^{-3} h^{3 / 2} M^{-2 / d} \leq$ 2 ,

$$
\sup _{x \in \mathbb{R}^{d}, k \in \llbracket 0, N \rrbracket}\left|u\left(t_{k}, x\right)-\bar{u}_{\delta}\left(t_{k}, x\right)\right|^{2} \leq G_{7.2} \mathcal{E}^{2} \text { (global). }
$$

As already explained in Subsection 3.3 the condition $\mathcal{B}$ (gradient) $\leq 2$ seems to be useless in the numerical example given in Subsection 4.2, since the observed error is still of order one w.r.t. $h$ although $\delta$ is of the same order as $h$ and $M$ is small. Moreover, in this example, the shape functions we use are Lagrange kernels and not $B$-splines: the above choice for $\Phi$ may not be justified from a numerical point of view.

Proof. The main steps of the proof of Theorem 3.2 still hold in this new frame. Anyhow, several differences are to be quoted.

First, the drift of the approximate transition is not bounded anymore, since it now depends on $\hat{v}$. Following the proof of Proposition 5.5. we can establish that the supremum norm of $\hat{v}$ is bounded by $C h^{-1 / 2}$. As a consequence, along an interval 
of the form $\left(t_{k}, t_{k+1}\right)$ for $\left.k \in \llbracket 0, N\right)$, the variation of $X$, given by (6.2), is still of order $h^{1 / 2}$. Hence, Lemma 6.2 is still true, and we can apply our strategy.

Second, in the proof of Theorem 6.1, Step One, the functional $F$ takes the form $F\left(s, x, x^{\prime}, y, p, A\right)=(1 / 2) \operatorname{tr}\left(A H_{u}(s, x)\right)+\nabla_{x} u(s, x) b\left(x^{\prime}, y, p\right), s \in[0, T], x, x^{\prime}, p \in$ $\mathbb{R}^{d}, y \in \mathbb{R}$ and $A \in \mathbb{R}^{d \times d}$. The term to investigate in (6.7) is now, on the event $\left\{\chi_{k+1}^{(i)}=1\right\}$ for $\left.i \in \llbracket 0,4^{d}\right)$ and on the interval $\left(t_{k}, t_{k+1}\right)$ for $\left.k \in \llbracket 0, N\right)$,

$$
\begin{aligned}
& F\left(s, X_{s}^{(i)}, X_{t_{k}+}^{(i)}, \bar{u}\left(t_{k+1}, X_{t_{k}+}^{(i)}\right), \hat{v}\left(t_{k}, X_{t_{k}+}^{(i)}\right), \sigma_{k}^{(i)} \gamma^{*} \gamma\left(\sigma_{k}^{(i)}\right)^{*}\right) \\
& \quad-F\left(s, X_{s}^{(i)}, X_{s}^{(i)}, \bar{V}_{s}^{(i)}, \bar{W}_{s}^{(i)}, \bar{\sigma}_{s}^{(i)}\left(\bar{\sigma}_{s}^{(i)}\right)^{*}\right) .
\end{aligned}
$$

Then, we let the reader check that the estimates for $D(1)$ and $D(2)$ in Lemma 6.3 involve a new term that refers to the difference between $\hat{v}$ and $v$. Now, Lemma 6.3 may be expressed with $C h \sum_{k=0}^{N-1} \mathbb{E}\left[\left(|\hat{v}-\bar{v}|^{2}\right)_{\delta}\left(t_{k}, X_{t_{k}}\right)\right]$ in addition to the right hand side. This leads to a new version for Theorem 6.1 .

Theorem 7.3. There exists a constant $C_{7.3}>0$ such that:

$$
\begin{aligned}
& \left|\left(\bar{u}_{\delta}-u\right)(0, x)\right|^{2}+C_{7.3}^{-1} h \sum_{k=1}^{N} \mathbb{E}\left[\left(|\bar{v}-v|^{2}\right)_{\delta}\left(t_{k-1}, X_{t_{k-1}}\right)\right] \\
& \quad \leq C_{7.3}\left[\mathcal{E}^{2} \text { (global) }+h \sum_{k=1}^{N}\left|\left(\bar{u}_{\delta}-u\right)\left(t_{k}, \cdot\right)\right|_{\infty}^{2}+h \sum_{k=0}^{N-1} \mathbb{E}\left[\left(|\hat{v}-\bar{v}|^{2}\right)_{\delta}\left(t_{k}, X_{t_{k}}\right)\right]\right] .
\end{aligned}
$$

Up to the modification of the initial condition, Theorem 7.3 together with the discrete version of Gronwall's lemma yield for all $k \in \llbracket 0, N)$

$$
\left|\left(\bar{u}_{\delta}-u\right)\left(t_{k}, \cdot\right)\right|_{\infty}^{2} \leq C \mathcal{E}^{2}(\text { global })+C h \sum_{j=k}^{N-1} \mathbb{E}\left[\left(|\bar{v}-\hat{v}|^{2}\right)_{\delta}\left(t_{j}, X_{t_{j}}\right)\right],
$$

for a constant $C$ independent of $k$ (whose value may vary in the sequel). Assume for the moment that for all $x \in \mathbb{R}^{d}$ and $\left.k \in \llbracket 0, N\right)$

$$
\begin{aligned}
& \left(|\hat{v}-\bar{v}|^{2}\right)_{\delta}\left(t_{k}, x\right) \leq C h \\
& \quad+C\left|\left(\bar{u}_{\delta}-u\right)\left(t_{k+1}, \cdot\right)\right|_{\infty}^{2}\left[1+\left(|\hat{v}|^{2}\right)_{\delta}\left(t_{k}, x\right)\right] \mathcal{B}^{2} \text { (gradient) }
\end{aligned}
$$

We can also write:

$$
\begin{aligned}
\left(|\hat{v}-\bar{v}|^{2}\right)_{\delta}\left(t_{k}, x\right) \leq & C h+C\left|\left(\bar{u}_{\delta}-u\right)\left(t_{k+1}, \cdot\right)\right|_{\infty}^{2}\left(|\hat{v}-\bar{v}|^{2}\right)_{\delta}\left(t_{k}, x\right) \mathcal{B}^{2} \text { (gradient) } \\
& +C\left|\left(\bar{u}_{\delta}-u\right)\left(t_{k+1}, \cdot\right)\right|_{\infty}^{2}\left[1+\left(|\bar{v}|^{2}\right)_{\delta}\left(t_{k}, x\right)\right] \mathcal{B}^{2} \text { (gradient) }
\end{aligned}
$$

For $\mathcal{B}$ (gradient) $\leq 2$, we obtain for all $x \in \mathbb{R}^{d}$ and $\left.k \in \llbracket 0, N\right)$

$$
\begin{aligned}
\left(|\hat{v}-\bar{v}|^{2}\right)_{\delta}\left(t_{k}, x\right) \leq & C h+4 C\left|\left(\bar{u}_{\delta}-u\right)\left(t_{k+1}, \cdot\right)\right|_{\infty}^{2}\left(|\hat{v}-\bar{v}|^{2}\right)_{\delta}\left(t_{k}, x\right) \\
& +4 C\left|\left(\bar{u}_{\delta}-u\right)\left(t_{k+1}, \cdot\right)\right|_{\infty}^{2}\left[1+\left(|\bar{v}|^{2}\right)_{\delta}\left(t_{k}, x\right)\right] .
\end{aligned}
$$

We complete the proof by the following lemma:

Lemma 7.4. With $C$ as in (7.4), assume that

$$
\left(C+2 C^{2}\right) \mathcal{E}^{2} \text { (global) } \exp \left(8 C^{2} T+8 C^{2} C(5.5) \leq 1 /(8 C) .\right.
$$

Then, for all $k \in \llbracket 0, N \rrbracket$,

$$
\left|\left(\bar{u}_{\delta}-u\right)\left(t_{k}, \cdot\right)\right|_{\infty}^{2} \leq\left(C+2 C^{2}\right) \mathcal{E}^{2} \text { (global) } \exp \left(8 C^{2} T+8 C^{2} C_{5.5}\right) .
$$

We then deduce Theorem 7.2 from Lemma 7.4 
Proof of Lemma 7.4. Inequality (7.5) clearly holds for $k=N$. Assume that it is true at given ranks $k+1, k+2, \ldots, N, k \in \llbracket 0, N)$, and prove that it holds at rank $k$. Due to (7.4) and the assumed bound for $\mathcal{E}$ (global), we claim for all $j \in \llbracket k, N)$ and $x \in \mathbb{R}^{d}$ :

$$
\begin{aligned}
\left(|\hat{v}-\bar{v}|^{2}\right)_{\delta}\left(t_{j}, x\right) \leq & C h+(1 / 2)\left(|\hat{v}-\bar{v}|^{2}\right)_{\delta}\left(t_{j}, x\right) \\
& +4 C\left|\left(\bar{u}_{\delta}-u\right)\left(t_{j+1}, \cdot\right)\right|_{\infty}^{2}\left[1+\left(|\bar{v}|^{2}\right)_{\delta}\left(t_{j}, x\right)\right],
\end{aligned}
$$

so that,

$$
\left(|\hat{v}-\bar{v}|^{2}\right)_{\delta}\left(t_{j}, x\right) \leq 2 C h+8 C\left|\left(\bar{u}_{\delta}-u\right)\left(t_{j+1}, \cdot\right)\right|_{\infty}^{2}\left[1+\left(|\bar{v}|^{2}\right)_{\delta}\left(t_{j}, x\right)\right] .
$$

Plug (7.6) into (7.2):

$$
\begin{aligned}
& \left|\left(\bar{u}_{\delta}-u\right)\left(t_{k}, \cdot\right)\right|_{\infty}^{2} \\
& \leq C \mathcal{E}^{2} \text { (global) } \\
& \quad+C h \sum_{j=k}^{N-1}\left\{2 C h+8 C\left|\left(\bar{u}_{\delta}-u\right)\left(t_{j+1}, \cdot\right)\right|_{\infty}^{2}\left[1+\mathbb{E}\left[\left(|\bar{v}|^{2}\right)_{\delta}\left(t_{j}, X_{t_{j}}\right)\right]\right]\right\} \\
& \leq\left(C+2 C^{2}\right) \mathcal{E}^{2}(\text { global }) \\
& \quad+8 C^{2} h \sum_{j=k}^{N-1}\left|\left(\bar{u}_{\delta}-u\right)\left(t_{j+1}, \cdot\right)\right|_{\infty}^{2}\left[1+\mathbb{E}\left[\left(|\bar{v}|^{2}\right)_{\delta}\left(t_{j}, X_{t_{j}}\right)\right]\right] .
\end{aligned}
$$

The discrete version of Gronwall's Lemma and Proposition 5.5 yield the result.

Proof of (7.3). For $x \in \mathcal{C}_{\infty}$, the very definitions of $\hat{v}$ and $\bar{v}$ (see Algorithm 3.1 and (7.1) ) give:

$$
\begin{aligned}
(\hat{v}-\bar{v})\left(t_{k}, x\right) \\
=h^{-1} \mathbb{E}\left[\left(\bar{u}_{\delta}\left(t_{k+1}, x+\mathcal{T}^{0}\left(t_{k}, x\right)\right)-\bar{u}_{\delta}\left(t_{k+1}, x+\mathcal{T}\left(t_{k}, x\right)\right)\right) g\left(\Delta B^{k}\right)^{*}\right] \gamma \\
=h^{-1} \mathbb{E}\left[\left(\left(\bar{u}_{\delta}-u\right)\left(t_{k+1}, x+\mathcal{T}^{0}\left(t_{k}, x\right)\right)\right.\right. \\
\left.\left.\quad-\left(\bar{u}_{\delta}-u\right)\left(t_{k+1}, x+\mathcal{T}\left(t_{k}, x\right)\right)\right) g\left(\Delta B^{k}\right)^{*}\right] \gamma \\
\quad+h^{-1} \mathbb{E}\left[\left(u\left(t_{k+1}, x+\mathcal{T}^{0}\left(t_{k}, x\right)\right)-u\left(t_{k+1}, x+\mathcal{T}\left(t_{k}, x\right)\right)\right) g\left(\Delta B^{k}\right)^{*}\right] \gamma \\
\equiv G(1, x)+G(2, x) .
\end{aligned}
$$

Start with $G(1, x)$ :

$$
\begin{aligned}
G(1, x)=-\mathbb{E} & {\left[\int _ { 0 } ^ { 1 } \left[\nabla_{x}\left(\bar{u}_{\delta}-u\right)\left(t_{k+1}, x+\lambda \beta\left(t_{k}, x\right) h+\Sigma\left(t_{k}, x\right) \gamma^{*} g\left(\Delta B^{k}\right)\right)\right.\right.} \\
& \left.\left.\times \beta\left(t_{k}, x\right) g\left(\Delta B^{k}\right)^{*}\right] d \lambda\right] \gamma .
\end{aligned}
$$

Admit for the moment the following lemma:

Lemma 7.5. There exists a constant $C_{7.5}$ such that for every $\ell \in \llbracket 1, d \rrbracket$ and for every bounded function $\varphi \in C^{2}\left(\mathbb{R}^{d}, \mathbb{R}\right)$ with bounded derivatives of order one and two and with Lipschitz continuous second order derivatives,

$$
\begin{aligned}
& \left|\mathbb{E}\left[\frac{\partial \varphi}{\partial x_{\ell}}\left(g\left(\Delta B^{k}\right)\right) g\left(\Delta B^{k}\right)^{*}\right]\right| \\
& \quad \leq C_{7.5}\left[|\varphi|_{\infty}+h M^{-2 / d}\left|\nabla^{(2)} \varphi\right|_{\infty}+h^{3 / 2} M^{-2 / d}\left|\nabla^{(3)} \varphi\right|_{\infty}\right] .
\end{aligned}
$$


Apply Lemma 7.5 to $G(1, x)$ or more specifically to the function $y \in \mathbb{R}^{d} \mapsto$ $\left(\bar{u}_{\delta}-u\right)\left(t_{k+1}, x+\lambda \beta\left(t_{k}, x\right) h+\Sigma\left(t_{k}, x\right) \gamma^{*} y\right) \beta_{i}\left(t_{k}, x\right)$, for $i \in \llbracket 1, d \rrbracket$ and $\lambda \in(0,1)$. The function $\left(\bar{u}_{\delta}-u\right)\left(t_{k+1}, \cdot\right)$ belongs to $\mathcal{C}^{2}\left(\mathbb{R}^{d}, \mathbb{R}\right)$ with bounded and Lipschitz continuous derivatives of order one and two, and for $i \in \llbracket 2,3 \rrbracket,\left|\nabla^{(i)}\left(\bar{u}_{\delta}-u\right)\left(t_{k+1}, \cdot\right)\right|_{\infty} \leq$ $C\left|\left(\bar{u}_{\delta}-u\right)\left(t_{k+1}, \cdot\right)\right|_{\infty} \delta^{-i}$. We deduce

$$
|G(1, x)| \leq C\left|\left(\bar{u}_{\delta}-u\right)\left(t_{k+1}, \cdot\right)\right|_{\infty}\left(1+\left|\hat{v}\left(t_{k}, x\right)\right|\right) \mathcal{B} \text { (gradient). }
$$

Now turn to $G(2, x)$. Expand it as $G(1, x)$ in (7.8) and subtract $\nabla_{x} u\left(t_{k+1}, x+\right.$ $\left.\lambda \beta\left(t_{k}, x\right) h\right)$ to $\nabla_{x} u\left(t_{k+1}, x+\lambda \beta\left(t_{k}, x\right) h+\Sigma\left(t_{k}, x\right) \gamma^{*} g\left(\Delta B^{k}\right)\right)$ (recall that $g\left(\Delta B^{k}\right.$ ) is centered). We obtain $|G(2, x)| \leq C h\left|\beta\left(t_{k}, x\right)\right| \leq C h^{1 / 2}$ since $\left|\beta\left(t_{k}, x\right)\right| \leq C h^{-1 / 2}$ (see Proposition (5.5). Thanks to (7.7) and (7.9), we derive (7.3).

Proof of Lemma 7.5 .

$$
\begin{aligned}
& \mathbb{E}\left[\frac{\partial \varphi}{\partial x_{\ell}}\left(g\left(\Delta B^{k}\right)\right) g\left(\Delta B^{k}\right)^{*}\right] \stackrel{\sqrt{3.5}}{=} \mathbb{E}\left[\frac{\partial \varphi}{\partial x_{\ell}}\left(g\left(\Delta B^{k}\right)\right)\left(\Delta B^{k}\right)^{*}\right] \\
& =\mathbb{E}\left[\left(\frac{\partial \varphi}{\partial x_{\ell}}\left(g\left(\Delta B^{k}\right)\right)-\frac{\partial \varphi}{\partial x_{\ell}}\left(\Delta B^{k}\right)\right)\left(\Delta B^{k}\right)^{*}\right]+\mathbb{E}\left[\frac{\partial \varphi}{\partial x_{\ell}}\left(\Delta B^{k}\right)\left(\Delta B^{k}\right)^{*}\right] \\
& \equiv \Phi(1)+\Phi(2) .
\end{aligned}
$$

First we investigate $\Phi(1)$ :

$$
\begin{aligned}
\Phi(1)= & \mathbb{E}\left[\left(\frac{\partial \varphi}{\partial x_{\ell}}\left(g\left(\Delta B^{k}\right)\right)-\frac{\partial \varphi}{\partial x_{\ell}}\left(\Delta B^{k}\right)\right)\left(\left(\Delta B^{k}\right)^{*}-g\left(\Delta B^{k}\right)^{*}\right)\right] \\
& +\mathbb{E}\left[\left(\frac{\partial \varphi}{\partial x_{\ell}}\left(g\left(\Delta B^{k}\right)\right)-\frac{\partial \varphi}{\partial x_{\ell}}\left(\Delta B^{k}\right)\right) g\left(\Delta B^{k}\right)^{*}\right] \\
= & \mathbb{E}\left[\left(\frac{\partial \varphi}{\partial x_{\ell}}\left(g\left(\Delta B^{k}\right)\right)-\frac{\partial \varphi}{\partial x_{\ell}}\left(\Delta B^{k}\right)\right)\left(\left(\Delta B^{k}\right)^{*}-g\left(\Delta B^{k}\right)^{*}\right)\right] \\
- & \int_{0}^{1}\left[\nabla_{x}\left(\frac{\partial \varphi}{\partial x_{\ell}}\right)\left(g\left(\Delta B^{k}\right)+\lambda\left(\Delta B^{k}-g\left(\Delta B^{k}\right)\right)\right)\right. \\
& \left.\times\left(\Delta B^{k}-g\left(\Delta B^{k}\right)\right) g\left(\Delta B^{k}\right)^{*}\right] d \lambda \\
= & \mathbb{E}\left[\left(\frac{\partial \varphi}{\partial x_{\ell}}\left(g\left(\Delta B^{k}\right)\right)-\frac{\partial \varphi}{\partial x_{\ell}}\left(\Delta B^{k}\right)\right)\left(\left(\Delta B^{k}\right)^{*}-g\left(\Delta B^{k}\right)^{*}\right)\right] \\
- & \int_{0}^{1}\left[\left(\nabla_{x}\left(\frac{\partial \varphi}{\partial x_{\ell}}\right)\left(g\left(\Delta B^{k}\right)+\lambda\left(\Delta B^{k}-g\left(\Delta B^{k}\right)\right)\right)-\nabla_{x}\left(\frac{\partial \varphi}{\partial x_{\ell}}\right)\left(g\left(\Delta B^{k}\right)\right)\right)\right. \\
& \left.\times\left(\Delta B^{k}-g\left(\Delta B^{k}\right)\right) g\left(\Delta B^{k}\right)^{*}\right] d \lambda \\
\equiv & \Phi(1,1)+\Phi(1,2),
\end{aligned}
$$

using (3.5) to obtain $\Phi(1,2)$. Now, we can use (3.4) to treat $\Phi(1,1)$. For $\Phi(1,2)$, we use the following result: as explained in the proof of Lemma 6.3, the square Gaussian quantization is still rate optimal in $L^{\beta}(\mathbb{P})$ for $\beta \in[2, d+2)$, that is, $\left(\mathbb{E}\left[\left|g\left(\Delta B^{k}\right)-\Delta B^{k}\right|^{\beta}\right]\right)^{\beta^{-1}} \leq C h^{1 / 2} M^{-1 / d}$; see [11]. Hence, we can deduce $|\Phi(1)| \leq$ $C\left(\left|\nabla^{(2)} \varphi\right|_{\infty}+\left|\nabla^{(3)} \varphi\right|_{\infty} h^{1 / 2}\right) h \times M^{-2 / d}$. Deal finally, for $j \in \llbracket 1, d \rrbracket$, with the $j^{\text {th }}$ coordinate of $\Phi(2)$ :

$$
\begin{aligned}
\Phi_{j}(2) & =(2 \pi)^{-d / 2} h^{1 / 2} \int_{\mathbb{R}^{d}} \frac{\partial \varphi}{\partial y_{\ell}}\left(h^{1 / 2} y\right) y_{j} \exp \left(-|y|^{2} / 2\right) d y \\
& =-(2 \pi)^{-d / 2} \int_{\mathbb{R}^{d}} \varphi\left(h^{1 / 2} y\right) \frac{\partial}{\partial y_{\ell}}\left(y_{j} \exp \left(-|y|^{2} / 2\right)\right) d y .
\end{aligned}
$$

Hence, $|\Phi(2)| \leq C|\varphi|_{\infty}$. This completes the proof. 


\section{REFERENCES}

1. F. Antonelli, Backward-Forward Stochastic Differential Equations, Ann. Appl. Prob. 3-3 (1993), 777-793. MR1233625 (95a:60079)

2. V. Bally, G. Pagès, and J. Printems, A quantization tree method for pricing and hedging multidimensional American options, Math. Finance 15 (2005), 119-168. MR.2116799(2005k:91142)

3. C. Bender and J. Zhang, Time discretization and Markovian iteration for coupled FBSDEs, Technical report, Weierstrass Institute, Berlin, and University of Southern California, Los Angeles (2006).

4. B. Bouchard and N. Touzi, Discrete time approximation and Monte-Carlo simulation of Backward Stochastic Differential Equations, Stoch. Proc. Appl. 111 (2004), 175-206. MR2056536 (2005b:65007)

5. S. Brenner and L. Scott, The Mathematical Theory of Finite Element Methods, Second edition. Texts in Applied Mathematics, Springer-Verlag, New York, 2002. MR1894376 (2003a:65103)

6. C. de Boor, A practical guide to splines. revised edition, Springer-Verlag, New York, 2001. MR 1900298 (2003f:41001)

7. F. Delarue, On the existence and uniqueness of solutions to FBSDEs in a non-degenerate case, Stoch. Proc. Appl. 99 (2002), 209-286. MR1901154 (2003c:60108)

8. F. Delarue and G. Guatteri, Weak existence and uniqueness for FBSDEs, Stoch. Proc. Appl. 116 (2006), 1712-1742. MR2307056

9. F. Delarue and S. Menozzi, A Forward Backward Stochatic Algorithm for Quasilinear PDEs, Ann. Appl. Prob. 16-1 (2006), 140-184. MR2209339 (2006m:60096)

10. J. Douglas, J. Ma, and P. Protter, Numerical methods for Forward-Backward Stochastic Differential Equations, Ann. Appl. Prob. 6 (1996), 940-968. MR1410123 (97k:60160)

11. S. Graf, H. Luschgy, and G. Pagès, Distortion mismatch in the quantization of probability measures, Technical report, no 1051, Laboratoire PMA, Universités Paris 6 et 7 (2006), http://hal.archives-ouvertes.fr/ccsd-00019228/en/.

12. S. Graf and H. Lushgy, Foundations of quantization for random vectors, LNM-1730, SpringerVerlag, 2000.

13. Y. Hu, P. Imkeller, and M. Müller, Utility maximization in incomplete markets, Ann. Appl. Prob. 15 (2005). MR2152241 (2006b:91071)

14. J. Jacod and A.N. Shiryaev, Limit theorems for stochastic processes, second edition, SpringerVerlag, 2004. MR.1943877(2003j:60001)

15. M. Kardar, G. Parisi, and Y.-C. Zhang, Dynamic scaling of growing interfaces, Phys. Rev. Lett. 56 (1986), 889-892.

16. M. Kobylanski, Backward stochastic differential equations and partial differential equations with quadratic growth, Ann. Prob. 28 (2000). MR1782267 (2001h:60110)

17. O.A. Ladyzhenskaya, V.A. Solonnikov, and N.N. Ural'ceva, Linear and quasilinear equations of parabolic type, Translations of Mathematical Monographs, Vol. 23, American Mathematical Society, Providence, 1967. MR0241822 (39:3159b)

18. J.P. Lemor, E. Gobet, and X. Warin, Rate of convergence of an empirical regression method for solving generalized backward stochastic differential equations, Bernoulli 12 (2006) no. 5, 889-916. MR2265667

19. _ A regression-based Monte-Carlo method to solve backward stochastic differential equations, Ann. Appl. Prob. 15 (2005), 2172-2002. MR2152657 (2006c:60078)

20. J. Ma, P. Protter, and J. Yong, Solving Forward-Backward Stochastic Differential Equations explicitly - a four step scheme, Prob. Th. Rel. Fields 98 (1994), 339-359. MR1262970 (94m:60118)

21. J. Ma and J. Yong, Forward-backward stochastic differential equations and their applications, LNM-1702, Springer-Verlag, 1999. MR1704232 (2000k:60118)

22. G. N. Milstein and M. V. Tretyakov, Numerical algorithms for semilinear parabolic equations with small parameter based on approximation of stochastic equations, Math. Comp. 69-229 (1999), 237-267. MR1653966 (2000i:65160)

23. — Discretization of forward-backward stochastic differential equations and related quasi-linear parabolic equations, IMA J Numer Anal 10.1093/imanum/drl019 (2006), http://imanum.oxfordjournals.org/cgi/content/abstract/drl019v1.

24. _ Numerical algorithms for forward-backward stochastic differential equations, SIAM J. Sci. Comp. 28 (2006), 561-582. MR2231721 
25. G.N. Milstein and M.V. Tretyakov, Stochastic numerics for mathematical physics, SpringerVerlag, Berlin, 2004. MR2069903 (2005f:60004)

26. E. Pardoux and S.G. Peng, Adapted solution of a Backward Stochastic Differential Equation, Systems Control Lett. 14-1 (1990), 55-61. MR1037747(91e:60171)

27. O. Rivière, Equations différentielles stochastiques progressives rétrogrades couplées: équations aux dérivées partielles et discrétisation, Ph.D. Thesis, Université Paris 5 René Descartes (2005).

28. A.N. Shiryaev, Probability, Second edition, Graduate Texts in Mathematics, 95, SpringerVerlag, New York, 1996. MR.1368405 (97c:60003)

29. W.A. Woyczyński, Burgers-KPZ turbulence, LNM-1700, Springer-Verlag, 1998. MR1732301 $(2000 \mathrm{j}: 60077)$

Université Paris 7, UfR de Mathématiques, Case 7012, 2, Place Jussieu, 75251 Paris Cedex 05, France

E-mail address: delarue@math.jussieu.fr

Université Paris 7, UfR de Mathématiques, Case 7012, 2, Place Jussieu, 75251 Paris Cedex 05, France

E-mail address: menozzi@math.jussieu.fr 